\title{
Hamiltonian constraints and unfree gauge symmetry
}

\author{
V. A. Abakumova $\odot^{*}$ and S. L. Lyakhovich $\odot^{\dagger}$ \\ Physics Faculty, Tomsk State University, Tomsk 634050, Russia
}

(Received 14 September 2020; accepted 30 October 2020; published 1 December 2020)

\begin{abstract}
We study Hamiltonian form of unfree gauge symmetry where the gauge parameters have to obey differential equations. We consider the general case such that the Dirac-Bergmann algorithm does not necessarily terminate at secondary constraints, and tertiary and higher order constraints may arise. Given the involution relations for the first-class constraints of all generations, we provide explicit formulas for unfree gauge transformations in the Hamiltonian form, including the differential equations constraining gauge parameters. All the field theories with unfree gauge symmetry share the common feature: they admit sort of "global constants of motion" such that do not depend on the local degrees of freedom. The simplest example is the cosmological constant in the unimodular gravity. We consider these constants as modular parameters rather than conserved quantities. We provide a systematic way of identifying all the modular parameters. We demonstrate that the modular parameters contribute to the Hamiltonian constraints, while they are not explicitly involved in the action. The Hamiltonian analysis of the unfree gauge symmetry is precessed by a brief exposition for the Lagrangian analogue, including explicitly covariant formula for degrees of freedom number count. We also adjust the Batalin-Fradkin-Vilkovisky-Becchi-Rouet-Stora-Tyutin Hamiltonian quantization method for the case of unfree gauge symmetry. The main distinction is in the content of the nonminimal sector and gauge fixing procedure. The general formalism is exemplified by traceless tensor fields of irreducible spin $s$ with the gauge symmetry parameters obeying transversality equations.
\end{abstract}

DOI: 10.1103/PhysRevD.102.125003

\section{INTRODUCTION}

Gauge symmetry is usually understood as a set of the infinitesimal transformations of the fields such that leaves the action intact, while the transformation parameters are the functions of space-time. Gauge symmetry is said unfree if the invariance of the action requires the gauge parameters to obey the system of partial differential equations. The general solution of the equations constraining gauge parameters must involve arbitrary functions of all $d$ space-time coordinates. If the solution includes arbitrary functions of $d-1$ coordinates or less, then this is not gauge symmetry. If no equations are imposed on the gauge parameters, they are all arbitrary functions.

The most known example of an unfree gauge symmetry is the volume-preserving diffeomorphism of unimodular gravity (UG). Various analogues of the linearized UG [1,2] are known among the free higher spin field theories, with gauge parameters constrained by transversality equations

\footnotetext{
abakumova@phys.tsu.ru

sll@phys.tsu.ru
}

Published by the American Physical Society under the terms of the Creative Commons Attribution 4.0 International license. Further distribution of this work must maintain attribution to the author(s) and the published article's title, journal citation, and DOI. Funded by SCOAP ${ }^{3}$.
$[3,4]$. The key distinction of UG from general relativity (GR) with $\Lambda$-term is that $\Lambda$ is a specific constant fixed from the outset in the action of GR, while UG comprises dynamics with any cosmological constant. For discussion of the role of cosmological constant in UG and further references, we cite [5]. Also, modifications of UG can be found in [6,7], where $\Lambda$ is defined dynamically, not as prefixed parameter in the action. All the field theories with unfree gauge symmetry share the common feature: they admit the "global constants of motion" such that do not depend on the local degrees of freedom, with $\Lambda$ of the UG being the simplest example. This general fact is explained from various viewpoints in the recent papers [8-10]. As the specific values of these integration constants are defined by the field asymptotics, not the Cauchy data, we consider them as modular parameters rather than conserved quantities. In the higher spin field analogues of UG, e.g., similar modular parameters exist, and their number grows with spin, although this fact has not previously been noticed.

While the examples of unfree gauge symmetry have been known for a long time, the general theory of this class of gauge systems began to develop relatively recently. In the paper [8], general structure is established for unfree gauge symmetry algebra in Lagrangian formalism, and the modification is proposed for the Faddeev-Popov (FP) quantization method such that accounts for the constraints imposed on gauge parameters. In the paper [9], the 
BV-BRST ${ }^{1}$ field-antifield formalism is worked out for the systems with unfree gauge symmetry. In the paper [10], the structures are identified in the algebra of Hamiltonian constraints such that describe unfree gauge symmetry. Before this work, the equations constraining gauge parameters in Hamiltonian formalism have been unknown even in specific models. The brief consideration of the paper [10] assumes that the Dirac-Bergmann algorithm terminates at secondary constraints, no tertiary ones are allowed. In this work, we provide systematic Hamiltonian description of unfree gauge symmetry in the general case, with the sequence of constraints of any finite order. Besides the reason of generality, this is also motivated by specific models. While in UG, the Dirac-Bergmann algorithm terminates at the stage of secondary constraints, in the higher spin field theories with unfree gauge symmetry, the sequence of constraints turns out linearly growing with spin, so the tertiary constraints arise for $s=3$. The number of modular parameters also grows with spin, and they all contribute to the constraints. The new phenomenon here is that the modular parameters, being connected to the nontrivial asymptotics of the fields, can make the constraints explicitly depending on the space-time point $x$, even though the original Lagrangian is $x$ independent. This phenomenon has previously unnoticed analogue in Lagrangian formalism.

The main goal of this paper is to work out Hamiltonian description of general unfree gauge symmetry. Then, we also extend the BFV-BRST ${ }^{2}$ formalism to this class of theories, with main modifications related to the nonminimal sector of ghosts. The general formalism is exemplified by the massless spin-s theory where the irreducible representation is realized by traceless tensors [3]. To make the paper self-contained, we precede the Hamiltonian description of unfree gauge symmetry with the corresponding Lagrangian formalism mostly providing the facts from $[8,9]$, with a more emphasis on modular parameters. We also provide a convenient formula for the degree of freedom counting in Lagrangian formalism in the case of unfree gauge symmetry.

\section{UNFREE GAUGE SYMMETRY IN LAGRANGIAN FORMALISM: COMPLETION FUNCTIONS, AND MODULAR PARAMETERS}

Unfree gauge symmetry is a deviation from the usual assumptions implied by general theory of gauge systems as it is formulated in the textbooks; see, e.g., [11]. This deviation has an impact on basic statements of gauge theory. Notice the second Noether theorem, which connects gauge symmetry of the action with Noether identities between Lagrangian equations. We can mention two assumptions implied by the theorem: (i) the gauge parameters are arbitrary functions of $x$ and (ii) any on-shell

\footnotetext{
${ }^{1}$ Batalin-Vilkovisky-Becchi-Rouet-Stora-Tyutin.

${ }^{2}$ Batalin-Fradkin-Vilkovisky-Becchi-Rouet-Stora-Tyutin.
}

vanishing local quantity ${ }^{3}$ reduces to a linear combination of the lhs of Lagrangian equations and their derivatives. The first assumption is obviously invalid once the symmetry is unfree. The second one is also inevitably violated for the case of unfree gauge symmetry as it is explained in the papers $[8,9]$. Let us rephrase the violation of the second assumption: the local quantities $\tau_{a}$ exist such that vanish on-shell, while they cannot be expanded in the lhs of Lagrangian equations with local coefficients,

$$
\exists \tau_{a}(\phi): \quad \tau_{a} \approx 0, \quad \tau_{a}(\phi) \neq K_{a}^{i}(\phi) \partial_{i} S .
$$

Here, we use the condensed notation. The condensed indices $a, i$ include space-time point $x$ and discrete labels. Summation over condensed indices includes integration over space-time, $\partial_{i} S(\phi)$ is a variational derivative of the action $S(\phi)$ by the field $\phi^{i}$, and the symbol $\approx$ means onshell equality. So, violation of (ii) means that ideal $I$ of onshell vanishing local quantities is not spanned by the lhs of Lagrangian equations $\partial_{i} S=0$. The local quantities $\tau \in I$ (1) are called completion functions. The generating set of ideal $I$ includes lhs of Lagrangian equations and a number of completion functions. In slightly different wording, any on-shell vanishing local quantity $T(\phi)$ is spanned off-shell by field equations and completion functions with the local expansion coefficients,

$$
T(\phi) \approx 0 \Leftrightarrow T(\phi)=T^{i}(\phi) \partial_{i} S(\phi)+T^{a}(\phi) \tau_{a}(\phi) .
$$

The identities can exist between the Lagrangian equations and completion functions,

$$
\Gamma_{\alpha}^{i}(\phi) \partial_{i} S(\phi)+\Gamma_{\alpha}^{a}(\phi) \tau_{a}(\phi) \equiv 0,
$$

where all the coefficients $\Gamma(\phi)$ are local. These relations can be understood as modification of the usual Noether identities for the case when the theory admits completion functions. Upon not quite restrictive regularity assumptions (see in $[8,9]$ ), the operators $\Gamma_{\alpha}^{a}(\phi)$, being the coefficients at completion functions, can admit at maximum a finite dimensional kernel,

$$
\begin{aligned}
\Gamma_{\alpha}^{a}(\phi) u_{a} & =0 \Rightarrow u_{a} \in M=\operatorname{Ker} \Gamma_{\alpha}^{a}, \\
\operatorname{dim} M & =n \in \mathbb{N} .
\end{aligned}
$$

The kernel $M$ is understood as a moduli space of the field theory. Elements of $M$ are parametrized by finite number of constant parameters $\Lambda$. Being parametrized by constants, the elements of $M$ can explicitly depend on the space-time point $x$. From the viewpoint of modified Noether identities (3), the completion functions $\tau_{a}$ are defined modulo the kernel $M$ (4). Specific element of the kernel is defined by

\footnotetext{
${ }^{3}$ By local quantity, we mean the function of space-time coordinates, fields, and their derivatives of finite order.
} 
the asymptotics of the fields, as $\tau_{a}$ should vanish on-shell everywhere, including boundary. From this perspective, the existence of completion functions (1) can be considered as a consequence of modified Noether identities (3) rather than a cause. Once the kernel of $\Gamma_{\alpha}^{a}$ is finite, the identities (3) mean that the local quantities $\tau_{a}$ reduce on-shell to a specific $\Lambda$-dependent function of $x$. This function can be subtracted from $\tau$, so the completion functions vanish onshell. On the other hand, $\Gamma_{\alpha}^{a}$ is a differential operator, and it does not have inverse in the class of differential operators, as the kernel exists. Once $\Gamma_{\alpha}^{a}$ is not locally invertible, completion function $\tau_{a}(\phi)$, being on-shell vanishing local quantity, cannot be expressed from the identities (3) as a linear combination of Lagrangian equations with local coefficients. In this sense, the identities (3) lead to existence of completion functions (1).

Be modified Noether identity (3) a consequence of existence of completion functions (1), or vice versa, anyway, it means that the action $S(\phi)$ enjoys unfree gauge symmetry. The unfree gauge transformation is defined by the coefficients $\Gamma_{\alpha}^{i}$ of the identities (3),

$$
\delta_{\varepsilon} \phi^{i}=\Gamma_{\alpha}^{i}(\phi) \varepsilon^{\alpha}
$$

while the operators $\Gamma_{\alpha}^{a}$ define the equations constraining gauge parameters,

$$
\Gamma_{\alpha}^{a}(\phi) \varepsilon^{\alpha}=0
$$

Let us mention the terminology: operators $\Gamma_{\alpha}^{i}$, being the coefficients at Lagrangian equations in modified Noether identities (3), are understood as unfree gauge symmetry generators, while $\Gamma_{\alpha}^{a}$, being the coefficients at the completion functions in (3), are considered as operators of gauge parameter constraints. Given the identities (3), the transformation (5) leaves the action intact off-shell once the parameters obey conditions (6),

$$
\delta_{\varepsilon} S(\phi) \equiv \partial_{i} S(\phi) \Gamma_{\alpha}^{i} \varepsilon^{\alpha} \equiv-\tau_{a} \Gamma_{\alpha}^{a} \varepsilon^{\alpha}=0 .
$$

In this way, we see that unfree gauge symmetry is a consequence of modified Noether identities (3). Proceeding from this observation first made in [8], we can find the Hamiltinian counterpart of the unfree gauge symmetry. It is sufficient to find the modified Noether identities (3) for the Hamiltonian equations with constraints, and the equations for gauge parameters (6) are immediately identified. This is done in the next section.

Let us briefly explain the modification of the FP ansatz needed to account for the unfree gauge symmetry. The modification is proposed in Ref. [8], where one can find a more detailed exposition of the method. In Sec. IV, we deduce this modified ansatz from the BFV-BRST formalism.
The ghosts assigned to the unfree gauge transformations (5) are assumed to obey equations

$$
\Gamma_{\alpha}^{a}(\varphi) C^{\alpha}=0, \quad \operatorname{gh}\left(C^{\alpha}\right)=1, \quad \epsilon\left(C^{\alpha}\right)=1,
$$

where $\Gamma_{\alpha}^{a}(\varphi)$ are the operators of gauge parameter constraints (6). Let us impose independent gauges $\chi^{I}(\phi)$. The index $I$ is condensed, so it includes the space coordinates $x^{\mu}$. The dimension of digital part of the index should be equal to the number of unconstrained gauge parameters. ${ }^{4}$ Once we use independent gauge-fixing conditions, the number of unfree gauge parameters will exceed the number of gauges, so FP matrix will be rectangular,

$$
\frac{\delta_{\varepsilon} \chi^{I}}{\delta \varepsilon^{\alpha}}=\Gamma_{\alpha}^{i}(\phi) \partial_{i} \chi^{I}(\phi) .
$$

Given the admissible gauge fixing conditions, the antighosts

$$
\bar{C}_{I}, \quad \operatorname{gh}\left(\bar{C}_{I}\right)=-1, \quad \epsilon\left(\bar{C}_{I}\right)=1
$$

are assigned to $\chi^{I}(\phi)$. The FP ansatz for path integral is adjusted to the case of unfree gauge symmetry in the following way:

$$
\begin{aligned}
& Z=\int[d \Phi] \exp \left\{\frac{i}{\hbar} S_{\mathrm{FP}}(\varphi)\right\}, \\
& \Phi=\left\{\phi^{i}, \pi_{I}, C^{\alpha}, \bar{C}_{I}, \bar{C}_{a}\right\}, \\
& \operatorname{gh}\left(\bar{C}_{a}\right)=-1, \quad \epsilon\left(\bar{C}_{a}\right)=1, \\
& \operatorname{gh}\left(\pi_{I}\right)=\epsilon\left(\pi_{I}\right)=0,
\end{aligned}
$$

where the FP action reads

$$
\begin{aligned}
S_{\mathrm{FP}}= & S(\phi)+\pi_{I} \chi^{I}(\phi) \\
& +\bar{C}_{I} \Gamma_{\alpha}^{i}(\phi) \partial_{i} \chi^{I}(\phi) C^{\alpha}+\bar{C}_{a} \Gamma_{\alpha}^{a}(\phi) C^{\alpha} .
\end{aligned}
$$

The Fourier multipliers $\bar{C}_{a}$ to the ghost constraints $\Gamma_{\alpha}^{a}(\phi) C^{\alpha}=0$ can be considered as antighosts, on equal footing with the antighosts $\bar{C}_{I}$ assigned to the gauge-fixing conditions $\chi^{I}(\phi)$. In Sec. IV, we shall see that these antighosts naturally arise from the Hamiltonian BFVBRST formalism.

Let us exemplify the above generalities about unfree gauge symmetry by the case of UG. Consider the unimodular metrics $g_{\mu \nu}(x)$, $\operatorname{det} g=-1$, in $d=4$. The usual explanations of gauge symmetry in UG proceed from the idea that the symmetry is a diffeomorphism consistent with unimodularity condition. This imposes the transversality

\footnotetext{
${ }^{4}$ In the next section, we explain the number of gauge conditions from the Hamiltonian perspective.
} 
equation on the parameter. We go another way, following the procedure above, and we shall see the same result.

Lagrangian equations of UG read

$$
\frac{\delta S[g]}{\delta g_{\mu \nu}} \equiv R^{\mu \nu}-\frac{1}{4} g^{\mu \nu} R \approx 0, \quad S=\int d^{4} x R .
$$

Taking divergence of the equations, and making use of Bianchi identity, we get

$$
\nabla_{\nu} \frac{\delta S[g]}{\delta g_{\mu \nu}} \equiv \nabla^{\mu} R \approx 0 .
$$

Unlike GR, the divergence of the field equations does not identically vanish. Once $\partial_{\mu} R \approx 0$, the scalar curvature is an on-shell constant, $R \approx \Lambda=$ const, where specific value of $\Lambda$ is defined by asymptotics of $g_{\mu \nu}$. So, we have the modular parameter $\Lambda$ and completion function $\tau \equiv R-\Lambda \approx 0$. Obviously, $\tau$ cannot be represented as linear combination of Eq. (14) and their derivatives, so it is a completion function indeed. Then, we get modified Noether identities (3) for UG,

$$
\nabla_{\nu} \frac{\delta S[g]}{\delta g_{\mu \nu}}-\nabla^{\mu} \tau \equiv 0
$$

This allows us to identify the unfree gauge symmetry transformations (5) and the gauge parameter constraints (6),

$$
\delta_{\varepsilon} g_{\mu \nu}=\nabla_{\mu} \varepsilon_{\nu}+\nabla_{\nu} \varepsilon_{\mu}, \quad \nabla_{\mu} \varepsilon^{\mu}=0 .
$$

We can also mention one more example of completion function noticed in literature concerning Maxwell-like higher spin field theory [4]. In this theory, the double divergence of the tracefull second-rank tensor vanishes onshell, $\partial_{\mu} \partial_{\nu} \varphi^{\mu \nu} \approx 0$, while it does not reduce to the lhs of the field equations and their derivatives. This fact is emphasized in the paper [12].

In the end of this section, we provide, without proof, a receipt for covariant degree of freedom (d.o.f) counting in the theories with unfree gauge symmetry. In so doing, we assume that the Lagrangian equations are involutive in the sense that they do not admit lower order differential consequences. The receipt can be deduced along the same lines as explained in the paper [13] for the gauge theories without constraints on gauge parameters.

The d.o.f. number is calculated as follows:

$$
N_{\text {d.o.f }}=n_{e} o_{e}-n_{s} o_{s}-n_{i} o_{i}+n_{c} o_{c},
$$

where $n_{e}, n_{s}, n_{i}$, and $n_{c}$ are the numbers, and $o_{e}, o_{s}, o_{i}, o_{c}$ are the orders of Lagrangian equations $\partial_{i} S=0$, gauge symmetry transformations $\delta_{\varepsilon} \varphi^{i}=\Gamma_{\alpha}^{i} \varepsilon^{\alpha}$, gauge identities $\Gamma_{\alpha}^{i} \partial_{i} S+\Gamma_{\alpha}^{a} \tau_{a}=0$, and constraints $\Gamma_{\alpha}^{a} \varepsilon^{\alpha}=0$, respectively. The order $o_{e}$ is defined by the highest order derivative in equations of motion (EoMs), $o_{s}$ is the order of gauge symmetry differential operator. The order of gauge identity, $o_{i}$, is a sum of $o_{s}$ and $o_{e}$, and $o_{c}$ is a sum of the order of constraint operator $\Gamma_{\alpha}^{a}$ and $o_{s}$.

Let us exemplify the d.o.f. number count (18) by the case of UG in $d=4$. We have nine equations of the second $\operatorname{order}(14), n_{e}=9, o_{e}=2$. There are four gauge symmetry transformations of the first order, and one first-order equation imposed on the gauge parameters (17), so $n_{s}=4, o_{s}=1, n_{c}=1, o_{c}=1+1=2$. There exist four gauge identities (16), $n_{i}=4$, of the third order $\left(o_{i}=1+2=3\right)$. So, according to (18), UG has 4 degrees of freedom by phase-space count, which corresponds to two "Lagrangian" d.o.f.

\section{CONSTRAINED HAMILTONIAN FORMALISM: HIGHER ORDER CONSTRAINTS, MODULAR PARAMETERS, AND UNFREE GAUGE SYMMETRY}

Any action functional can be brought to equivalent Hamiltonian form with primary constraints,

$$
\begin{aligned}
S & =\int d t\left(p_{i} \dot{q}^{i}-H_{T}(q, p, \lambda)\right), \\
H_{T}(q, p, \lambda) & =H(q, p)+\lambda^{\alpha_{1}} \stackrel{(1)}{T}_{\alpha_{1}}(q, p),
\end{aligned}
$$

where $q^{i}, p_{i}$ are canonical variables, and $\lambda^{\alpha_{1}}$ are Lagrange multipliers. All these variables can be viewed as the fields $\phi=(q, p, \lambda)$, and then we can apply the general consideration of the previous section to the action (19). As explained in the previous section, the unfree gauge symmetry (5), (6) is caused by modified Noether identities (3) which involve, besides the original Lagrangian equations $\partial_{i} S(\phi)=0$ and gauge generators $\Gamma_{\alpha}^{i}$, two more ingredients: completion functions $\tau_{a}(\phi)$ and operators of gauge parameter constraints $\Gamma_{\alpha}^{a}$. The key point in finding the unfree gauge symmetry of any action functional is to find a modified Noether identities (3) involving the operator $\Gamma_{\alpha}^{a}$ with a finite kernel (4). Once the identities are found, the coefficients at the equations define the gauge generators, while the operators $\Gamma_{\alpha}^{a}$ give the equations imposed on the gauge parameters. Hamiltonian action (19), due to the canonical structure, is very convenient for algorithmically deducing modified Noether identities (3). The idea is quite simple: we apply the Dirac-Bergmann algorithm of iterating constraints. We assume that no Lagrange multiplier is fixed, so all the constraints are first class. In the local field theory, the algorithm should terminate in a finite number of iterations. Termination of the algorithm is a (modified) Noether identity. This key fact is explained below in this section.

Once the modified Noether identities (3) are established, one can find the gauge transformation for the fields $\phi=$ $(q, p, \lambda)$ by identifying the coefficients at the corresponding 
equations, while the gauge parameter constraints are defined by the coefficient at the completion functions in the identity. As one can guess, the roles of completion functions are plaid in Hamiltonian formalism by secondary constraints of all generations. For the case when the sequence of constraints terminates at the secondary constraints, without tertiary and higher order ones, this program has been already implemented in the paper [10]. Here we consider the general case. When the secondary constraints lead to the higher order ones, and the involution coefficients include differential operators with finite kernel, this can lead, in general, to explicit dependence of kernel elements on space-time coordinates $x$. Through this mechanism, the explicit time dependence can arise in the higher order constraints even if the original action is translation invariant. The explicit $x$ dependence of secondary constraints is due to the field asymptotics which is defined by modular parameters.

Let us consider iteration of secondary constraints to deduce Hamiltonian form of identity (3) and get in this way the unfree gauge symmetry (5), (6) for Hamiltonian action (19). EoMs read

$$
\begin{gathered}
\frac{\delta S}{\delta p_{i}} \equiv \dot{q}^{i}-\left\{q^{i}, H_{T}(q, p, \lambda)\right\}=0 \\
\frac{\delta S}{\delta q^{i}} \equiv-\dot{p}_{i}+\left\{p_{i}, H_{T}(q, p, \lambda)\right\}=0 \\
\frac{\delta S}{\delta \lambda^{\alpha_{1}}} \equiv-{\stackrel{(1)}{\alpha_{1}}}_{1}(q, p)=0 .
\end{gathered}
$$

Following the Dirac-Bergmann algorithm, we take time derivative of primary constraints (21) and combine it with the evolutionary equations (20) to exclude the time derivatives. The result is at most linear in $\lambda$. As the multipliers remain indefinite, all the coefficients at $\lambda$ should be considered as on-shell vanishing, so the derivative of the primary constraints reduces to the combination of primary and secondary constraints,

$$
\begin{aligned}
\frac{d}{d t} \stackrel{(1)}{T}_{\alpha_{1}}(q, p)= & \left\{\stackrel{(1)}{T}_{\alpha_{1}}(q, p), H_{T}(q, p, \lambda)\right\} \\
= & V_{\alpha_{1}}^{\beta_{1}}(q, p, \lambda) \stackrel{(1)}{T} \beta_{\beta_{1}}(q, p) \\
& +\stackrel{(1)}{\Gamma}{\stackrel{\beta}{\alpha_{1}}}_{1}(q, p, \lambda) \stackrel{(2)}{T}_{\beta_{2}}(q, p) .
\end{aligned}
$$

Unfree gauge symmetry corresponds to the case when the structure coefficient $\stackrel{(1)}{\Gamma}_{\alpha_{1}}^{\beta_{2}}(q, p, \lambda)$ is a differential operator with finite kernel (4). This includes the case of zero kernel, while no inverse exists for $\stackrel{(1)}{\Gamma}$ in the class of differential operators. This has been first noticed in Ref. [10], though this paper assumed no higher order constraints appear.
Relation (22) defines secondary constraints $\stackrel{(2)}{T}$ modulo (1)

kernel of $\Gamma$. The kernel is parametrized by finite set of constant modular parameters $\Lambda$. The elements of the kernel can be specific $\Lambda$-dependent functions of space-time point $x$. The latter fact means that $\stackrel{(2)}{T}$ can be explicitly time dependent,

$$
\begin{aligned}
& \stackrel{(2)}{T} \beta_{2}(q, p, \Lambda, t)=T_{\beta_{2}}(q, p)+u_{\beta_{2}}(\Lambda, t, q, p), \\
& \stackrel{(1)}{\Gamma}{ }_{\alpha_{1}}^{\beta_{2}} u_{\beta_{2}}(\Lambda, t, q, p)=0 .
\end{aligned}
$$

Further examination of the stability of the secondary constraints has to account for the possible explicit time dependence which can originate from the kernel of $\stackrel{(1)}{\Gamma}$. The kernel depends, in its own turn, on the asymptotics of the fields.

Consider now the sequence of $n$ stability conditions of constraints labeled by index $k, k=2, \ldots, n$. The time derivatives of secondary constraints should vanish on-shell that leads to tertiary constraints, etc. Stability of the $l$-order constraints $\stackrel{(l)}{T}$ leads to $\stackrel{(l+1)}{T}$,

$$
\begin{aligned}
\frac{d}{d t} \stackrel{(l)}{T}_{\alpha_{l}}(q, p)= & \frac{\partial}{\partial t} \stackrel{(l)}{T}_{\alpha_{l}}(q, p)+\left\{\stackrel{(l)}{T} \alpha_{\alpha_{l}}(q, p), H_{T}(q, p, \lambda)\right\} \\
= & \sum_{m=1}^{l} \stackrel{(1)}{V}_{\alpha_{1}}^{\beta_{m}}(q, p, \lambda) \stackrel{(m)}{T} \beta_{\beta_{m}}(q, p) \\
& +\stackrel{(l)}{\Gamma}_{\alpha_{l}}^{\beta_{l+1}}(q, p, \lambda) \stackrel{(l+1)}{T}{ }_{\beta_{l+1}}(q, p),
\end{aligned}
$$

where $l=2, \ldots, n-1$. The coefficients $\stackrel{(l)}{\Gamma}$ at the constraints $(l+1)$

of next generation $T$ are the differential operators with a finite kernel. Therefore, constraints of $(l+1)$-st generation are defined modulo the kernel elements much like the secondary ones (23). In general, the kernel is different for different $l$ 's. The algorithm terminates when no further constraints appear,

$$
\begin{aligned}
\frac{d}{d t} \stackrel{(n)}{T}_{\alpha_{n}}(q, p) & =\frac{\partial}{\partial t} \stackrel{(n)}{T}_{\alpha_{n}}(q, p)+\left\{\stackrel{(n)}{T} \alpha_{\alpha_{n}}(q, p), H_{T}(q, p, \lambda)\right\} \\
& =\sum_{m=1}^{n} \stackrel{(n)}{V}_{\alpha_{n}}^{\beta_{m}}(q, p, \lambda) \stackrel{(m)}{T}_{\beta_{m}}(q, p)
\end{aligned}
$$

Note that constraints $\stackrel{(k)}{T}_{\alpha_{k}}, k=2, \ldots, n$ contain modular parameters defined by asymptotics of the field and can be explicitly time dependent. Once $\Gamma$ 's are differential operators, the secondary constraints of all generations (22), (24) are not differential consequences of original variational 
equations (20), (21), while they vanish on-shell, so they are completion functions (1).

Notice that all the structure functions $V, \Gamma$ in relations (22), (24), (25) are at most linear in $\lambda$, so it is useful to introduce separate notation for the coefficients at $\lambda$ 's and $\lambda$-independent terms,

$$
\stackrel{(r)}{V_{\alpha_{r}}^{\beta_{s}}}(q, p, \lambda)=V_{\alpha_{r}}^{\beta_{s}}(q, p)+U_{\alpha_{r} \gamma_{1}}^{\beta_{s}}(q, p) \lambda^{\gamma_{1}},
$$

where $r, s=1, \ldots, n$,

$$
\stackrel{(r) \beta_{r+1}}{\Gamma_{\alpha_{r}}}(q, p, \lambda)=\Gamma_{\alpha_{r}}^{\beta_{r+1}}(q, p)+U_{\alpha_{r} \gamma_{1}}^{\beta_{r+1}}(q, p) \lambda^{\gamma_{1}},
$$

where $r=1, \ldots, n, r+1 \leq n$.

Once the secondary constraints $\stackrel{(k)}{T}, k=2, \ldots, n$ of all generations play the role of completion functions (1), the relations of the Dirac-Bergmann algorithm (22), (24), (25) can be assembled into the modified Noether identities (3),

$$
\begin{aligned}
& \left\{\stackrel{(1)}{T}_{\alpha_{1}}, q^{i}\right\} \frac{\delta S}{\delta q^{i}}+\left\{\stackrel{(1)}{T}_{\alpha_{1}}, p_{i}\right\} \frac{\delta S}{\delta p_{i}} \\
& +\left(\delta_{\alpha_{1}}^{\beta_{1}} \frac{d}{d t}-V_{\alpha_{1}}^{\beta_{1}}(q, p, \lambda)\right) \frac{\delta S}{\delta \lambda^{\beta_{1}}} \\
& +\stackrel{(1)}{\Gamma}_{\alpha_{1}}^{\beta_{2}}(q, p, \lambda) \stackrel{(2)}{T_{\beta_{2}}} \equiv 0, \\
& \left\{\stackrel{(l)}{T}_{\alpha_{l}}, q^{i}\right\} \frac{\delta S}{\delta q^{i}}+\left\{\stackrel{(l)}{T}_{\alpha_{l}}, p_{i}\right\} \frac{\delta S}{\delta p_{i}}
\end{aligned}
$$

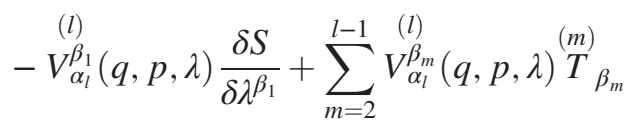

$$
\begin{aligned}
& +\left(-\delta_{\alpha_{l}}^{\beta_{l}} \frac{d}{d t}+{\stackrel{(l)}{\alpha_{\alpha_{l}}}}_{(q, p, \lambda)}\right)^{\beta_{\beta_{l}}} \\
& +\Gamma_{\alpha_{l}}^{\beta_{l+1}}(q, p, \lambda) \stackrel{(l+1)}{T}_{\beta_{l+1}}^{\beta_{l}} \equiv 0,
\end{aligned}
$$

where $l=2, \ldots, n-1$,

$$
\begin{aligned}
& \left\{\stackrel{(n)}{T}_{\alpha_{n}}, q^{i}\right\} \frac{\delta S}{\delta q^{i}}+\left\{\stackrel{(n)}{T} \alpha_{\alpha_{n}}, p_{i}\right\} \frac{\delta S}{\delta p_{i}} \\
& -V_{\alpha_{n}}^{\beta_{1}}(q, p, \lambda) \frac{\delta S}{\delta \lambda^{\beta_{1}}}+\sum_{l=2}^{n-1} V_{\alpha_{n}}^{\beta_{1}}(q, p, \lambda) \stackrel{(l)}{T_{\beta_{l}}} \\
& +\left(-\delta_{\alpha_{n}}^{\beta_{n}} \frac{d}{d t}+V_{\alpha_{n}}^{\beta_{\beta_{n}}}(q, p, \lambda)\right) \stackrel{(n)}{T}_{\beta_{n}} \equiv 0 .
\end{aligned}
$$

The coefficients at the variational equations in the identities (3) define unfree gauge variations (5) of corresponding variables, while the coefficients at completion functions define the constraints imposed on the gauge parameters (6). Given the modified Noether identities in the Hamiltonian form (28)-(30), with $q, p, \lambda$ being the fields, and the secondary constraints $\stackrel{(k)}{T}$ being the completion functions, we arrive at the Hamiltonian form of the unfree gauge symmetry,

$$
\begin{gathered}
\delta_{\varepsilon} O(q, p)=\sum_{r=1}^{n}\left\{O, \stackrel{(r)}{T}{ }_{\alpha_{r}}\right\} \varepsilon^{\alpha_{r}} \\
\delta_{\varepsilon} \lambda^{\alpha_{1}}=\left(\delta_{\beta_{1}}^{\alpha_{1}} \frac{d}{d t}+{\stackrel{(1)}{V_{\beta_{1}}^{\alpha_{1}}}}_{(q, p, \lambda)) \varepsilon^{\beta_{1}}}\right. \\
+\sum_{k=2}^{n} V_{\beta_{k}}^{\alpha_{1}}(q, p, \lambda) \varepsilon^{\beta_{k}}
\end{gathered}
$$

while equations constraining gauge parameters (6) read

$$
\begin{aligned}
& \left(\delta_{\beta_{l}}^{\alpha_{l}} \frac{d}{d t}+\stackrel{(l)}{V}_{\beta_{l}}^{\alpha_{l}}(q, p, \lambda)\right) \varepsilon^{\beta_{l}}+\sum_{m=l+1}^{n} \stackrel{(m)}{V} \underset{\beta_{m}}{\alpha_{l}}(q, p, \lambda) \varepsilon^{\beta_{m}} \\
& \quad+\stackrel{(l-1)}{\Gamma}{ }_{\beta_{l-1}}^{\alpha_{l-1}}(q, p, \lambda) \varepsilon^{\beta_{l-1}}=0
\end{aligned}
$$

where $l=2, \ldots, n-1$,

$$
\begin{aligned}
& \left(\delta_{\beta_{n}}^{\alpha_{n}} \frac{d}{d t}+{\stackrel{(n)}{V_{n}}}_{\alpha_{n}}^{\alpha_{n}}(q, p, \lambda)\right) \varepsilon^{\beta_{n}} \\
& \quad+\stackrel{(n-1)}{\Gamma} \underset{\beta_{n-1}}{\alpha_{n}}(q, p, \lambda) \varepsilon^{\beta_{n-1}}=0 .
\end{aligned}
$$

As one can see, the gauge transformations are generated by the constraints of all generations (31), (32), while corresponding gauge parameters are bound by the differential equations (33), (34). One can verify by direct computation that transformations (31), (32) leave original Hamiltonian action (19) intact. Given involution relations of Hamiltonian and constraints (22), (24), (25), the gauge variation (31), (32) of the action reads

$$
\begin{aligned}
\delta_{\varepsilon} S \equiv & \int d t\left\{\sum _ { l = 2 } ^ { n - 1 } \left[\left(\delta_{\beta_{l}}^{\alpha_{l}} \frac{d}{d t}+\stackrel{(l)}{V} \underset{\beta_{l}}{\alpha_{l}}\right) \varepsilon^{\beta_{l}}\right.\right. \\
& \left.+\sum_{m=l+1}^{n} \stackrel{(m)}{V}{ }_{\beta_{m}}^{\alpha_{l}} \varepsilon^{\beta_{m}}+\stackrel{(l-1)}{\Gamma} \underset{\beta_{l-1}}{\alpha_{l}} \varepsilon^{\beta_{l-1}}\right] \stackrel{(l)}{T_{\alpha_{l}}} \\
& +\left[\left(\delta_{\beta_{n}}^{\alpha_{n}} \frac{d}{d t}+\stackrel{(n)}{V}{ }_{\beta_{n}}^{\alpha_{n}}\right) \varepsilon^{\beta_{n}}+\stackrel{(n-1)}{\Gamma}{ }_{\beta_{n-1}}^{\alpha_{n}} \varepsilon^{\beta_{n-1}}\right] \stackrel{(n)}{T} \alpha_{\alpha_{n}} \\
& \left.-\frac{1}{2} \frac{d}{d t}\left(\sum_{r=1}^{n} \stackrel{(r)}{T}_{\alpha_{r}} \varepsilon^{\alpha_{r}}\right)\right\}=0 .
\end{aligned}
$$


By virtue of Eqs. (33) and (34), imposed on the gauge parameters, the integrand reduces to the total derivative.

Let us discuss the structure of Eqs. (33) and (34) constraining gauge parameters. To demonstrate key features of the equations, consider the toy model such that has only one constraint of each generation, so no indices $\alpha_{k}$ are needed. The next simplification is that all the constraints are assumed commuting. So, the involution relations (22), (24), (25) get a simple form,

$$
\begin{aligned}
& \{\stackrel{(1)}{T}, H\}=\stackrel{(1)}{\Gamma} \stackrel{(2)}{T},\{\stackrel{(l)}{T}, H\}=\stackrel{(l)}{\Gamma} \stackrel{(l+1)}{T}, \\
& \{\stackrel{(n)}{T}, H\}=0,\{\stackrel{(r)}{T}, \stackrel{(s)}{T}\}=0,
\end{aligned}
$$

where $l=2, \ldots, n-1 ; r, s=1, \ldots, n$. Given the involution relations, gauge transformations (31), (32) read

$$
\delta_{\epsilon} O=\sum_{r=1}^{n}\{O, \stackrel{(r)}{T}\} \varepsilon^{r}, \quad \delta_{\epsilon} \lambda=\dot{\varepsilon}^{1} .
$$

Equations (33) and (34) constraining gauge parameters $\varepsilon^{r}$ read

$$
\dot{\varepsilon}^{r+1}+\stackrel{(r)}{\Gamma} \varepsilon^{r}=0, \quad r=1, \ldots, n-1 .
$$

$$
(r)
$$

If operators $\Gamma, r=1, \ldots, n-1$, were all invertible in the class of differential operators, one could express all the gauge parameters $\varepsilon^{r}$ as the derivatives of the last one,

$$
\varepsilon^{r}=(\stackrel{(r)}{\Gamma})^{-1} \frac{d}{d t}(\stackrel{(r+1)}{\Gamma})^{-1} \ldots \frac{d}{d t}(\stackrel{(r-1)}{\Gamma})^{-1} \frac{d}{d t} \varepsilon^{n} .
$$

Relation (39) is a general solution for Eq. (38). Given the solution, one can substitute all the gauge parameters $\varepsilon^{r}$, $r=1, \ldots, n-1$, in terms of the unique unconstrained parameter $\varepsilon^{n}$, into the gauge transformation (31), (32). In this way, we arrive at the gauge transformation without constraints on gauge parameters but with higher derivatives of the unconstrained parameter. The most general case of this type, when the higher order gauge transformation generators can be constructed for the evolutionary equations with constraints, is considered in the paper [14]. The unfree gauge symmetry arises in the example above when at least one of operators $\Gamma$ in involution relations (36) does not admit inverse in the class of differential operators. Notice the special case of this type, when operators are nondegenerate, i.e., $\operatorname{ker} \Gamma=0$, while no $\Gamma^{-1}$ exists in the class of differential operators. As the example, we can mention the unimodular gravity with asymptotically flat metric. The role of $\Gamma_{\alpha}^{a}$ is plaid by partial derivative $\partial_{\mu}$, whose kernel is a constant. If the fields vanish at infinity, the kernel is zero, while no local inverse exists for the operator. In this case, the higher order unconstrained symmetry can exist, though it is reducible. For general linear field theories, the reducible higher order gauge symmetry, being equivalent to the unfree first-order symmetry, is described in Ref. [15] in Lagrangian formalism. The reducible unconstrained symmetry for this class of nonlinear theories will be considered elsewhere.

Let us mention that the number of equations (33), (34) imposed on the gauge parameters equals to the number of secondary constraints of all generations, while the number of gauge parameters is the number of constraints of all generations, including primary ones. All equations (33), (34) are independent; there are no identities among them, because every equation is resolved with respect to the derivative of a unique gauge parameter. Therefore, the number of independent gauge parameters equals to the number of primary constraints. If it was possible to locally express all the parameters in terms of independent ones and their derivatives, like in the example above, there would be $m_{1}$ independent gauge transformations, where $m_{1}$ is the number of primary constraints. On the other hand, corresponding number of time derivatives of $m_{1}$ independent gauge parameters $\varepsilon^{n}$ essentially contribute to the gauge transformations of dependent gauge parameters $\varepsilon^{r}$, $r=1, \ldots, n-1$. Therefore, overall $m$ independent parameters and their time derivatives would be involved in the gauge transformation (31), where $m$ is the total number of constraints. Hence, the on-shell gauge invariants should Poisson-commute on-shell with the constraints of all generations, even if the gauge symmetry is unfree. This would be true even if $m_{1}$ independent higher order gauge transformations cannot be explicitly extracted from $m$ unfree first-order transformations in the local way. Here, we do not provide a more rigorous justification of this observation, limiting ourselves to the explanations given above.

Once the unfree gauge symmetry corresponds to the higher order symmetry with $m_{1}$ independent parameters, it would be sufficient to impose $m_{1}$ independent gauge-fixing conditions. This number of required gauge conditions remain the same, even if the independent gauge parameters cannot be explicitly found from Eqs. (33) and (34) in the local form. If the gauges are imposed only on the phasespace variables, not Lagrange multipliers, then nondegeneracy condition of the gauges $\chi^{\alpha_{1}}$ reads

$$
\operatorname{rank}\left\{\chi^{\alpha_{1}}, T_{\beta}\right\}=m_{1},
$$

where $T_{\beta}$ stands for the complete set of all constraints, including primary, secondary, tertiary, etc., $\beta=\left(\beta_{1}, \ldots, \beta_{n}\right)$. Once the number is different of the constraints and gaugefixing conditions, the nonminimal ghost sector has to be modified in the BFV-BRST formalism for the case of unfree gauge symmetry. This issue is considered in the next section. 


\section{HAMILTONIAN BFV-BRST FORMALISM FOR UNFREE GAUGE SYMMETRY}

Construction of the formalism begins with introducing the minimal sector of ghosts. Once the on-shell gauge invariants for the unfree gauge symmetry are defined by the requirement to Poisson-commute on-shell with the constraints of all generations, the minimal sector is introduced along the same lines as for any first-class constrained system [11]. Every first-class constraint is assigned with canonical pair of ghosts with usual Grassmann parity and ghost number grading,

$$
\begin{aligned}
& \stackrel{(r)}{T}_{\alpha_{r}} \rightarrow\left\{C^{\alpha_{r}}, \bar{P}_{\beta_{r}}\right\}=\delta_{\beta_{r}}^{\alpha_{r}}, \\
& \operatorname{gh}\left(C^{\alpha_{r}}\right)=-\operatorname{gh}\left(\bar{P}_{\alpha_{r}}\right)=1, \\
& \epsilon\left(C^{\alpha_{r}}\right)=\epsilon\left(\bar{P}_{\alpha_{r}}\right)=1, \quad r=1, \ldots, n .
\end{aligned}
$$

The Hamiltonian BFV-BRST generator of minimal sector begins with the constraints,

$$
\begin{gathered}
Q_{\text {min }}=\sum_{r=1}^{n} C^{\alpha_{r}} \stackrel{(r)}{T}_{\alpha_{r}}+\ldots, \\
\operatorname{gh}\left(Q_{\text {min }}\right)=1, \quad \epsilon\left(Q_{\text {min }}\right)=1,
\end{gathered}
$$

where ... stands for $\bar{P}$-depending terms. These terms are iteratively defined by the equation

$$
\left\{Q_{\min }, Q_{\min }\right\}=0 .
$$

The ghost extension of the Hamiltonian begins with the original Hamiltonian $H$,

$$
\mathcal{H}=H+\ldots, \quad \operatorname{gh}(\mathcal{H})=0, \quad \epsilon(\mathcal{H})=0 .
$$

The specifics of the unfree gauge symmetry are that the completion functions (1), and hence the secondary constraints may depend on the space-time coordinates, even if the original Lagrangian is $x$ independent. The $x$ dependence of the constraints is connected with the asymptotics of the fields. Once the constraints involve time, the BRST generator $Q_{\min }$ can be explicitly time dependent. The explicit time dependence of $Q_{\min }$ results in appropriate modification [16] of the equation for $\mathcal{H}$,

$$
\frac{\partial}{\partial t} Q_{\min }+\left\{Q_{\min }, \mathcal{H}\right\}=0 .
$$

This equation defines the $\bar{P}$-dependent terms in $\mathcal{H}$. Equation (45) means that Hamiltonian $\mathcal{H}$ is not BRST invariant. This is a natural consequence of the relations (24) which mean that the original Hamiltonian is not invariant under the unfree gauge symmetry transformations (31), (33), (34). The Hamiltonian action (19), however, is gauge invariant, see (35). For a similar reason, the corresponding path integral is gauge invariant in the BRST-BFV formalism, even though the Hamiltonian $\mathcal{H}$, being a solution of Eq. (45), is not a BRST invariant. This fact is proven for general nonstationary constrained system in Ref. [16].

Consider now the nonminimal sector for the unfree gauge theory. Once the number of gauge fixing conditions coincides with the number of primary constraints, the same number of nonminimal sector ghosts is introduced,

$$
\begin{aligned}
\left\{P^{\alpha_{1}}, \bar{C}_{\beta_{1}}\right\} & =\delta_{\beta_{1}}^{\alpha_{1}}, \quad \operatorname{gh}\left(P^{\alpha_{1}}\right)=-\operatorname{gh}\left(\bar{C}_{\alpha_{1}}\right)=1, \\
\epsilon\left(P^{\alpha_{1}}\right) & =\epsilon\left(\bar{C}_{\alpha_{1}}\right)=1 .
\end{aligned}
$$

The Lagrange multiplier canonical pairs are introduced for primary constraints $\stackrel{(1)}{T}_{\alpha_{1}}$ and gauge fixing conditions $\chi^{\alpha_{1}}$,

$$
\begin{aligned}
\left\{\lambda^{\alpha_{1}}, \pi_{\beta_{1}}\right\} & =\delta_{\beta_{1}}^{\alpha_{1}}, \quad \operatorname{gh}\left(\lambda^{\alpha_{1}}\right)=\operatorname{gh}\left(\pi_{\alpha_{1}}\right)=0, \\
\epsilon\left(\lambda^{\alpha_{1}}\right) & =\epsilon\left(\pi_{\alpha_{1}}\right)=0 .
\end{aligned}
$$

Complete BRST generator extends the minimal sector one in the usual way,

$$
Q=Q_{\min }+\pi_{\alpha_{1}} P^{\alpha_{1}} .
$$

Gauge-fixing conditions involve the time derivative of Lagrange multiplier and the function of original phasespace variables,

$$
\dot{\lambda}^{\alpha_{1}}-\chi^{\alpha_{1}}(q, p)=0
$$

Given the gauge conditions, the gauge Fermion is introduced,

$$
\Psi=\bar{C}_{\alpha_{1}} \chi^{\alpha_{1}}+\lambda^{\alpha_{1}} \bar{P}_{\alpha_{1}},
$$

and gauge-fixed Hamiltonian is defined by the usual rule,

$$
H_{\Psi}=\mathcal{H}+\{Q, \Psi\}
$$

This Hamiltonian provides conservation of the BRST generator $Q$ much like $\mathcal{H}$. The gauge-fixed BFV-BRST action reads

$$
\begin{aligned}
S_{\mathrm{BRST}}^{\Psi}= & \int d t\left(p_{i} \dot{q}^{i}+\pi_{\alpha_{1}} \dot{\lambda}^{\alpha_{1}}\right. \\
& \left.+\sum_{r=1}^{n} \bar{P}_{\alpha_{r}} \dot{C}^{\alpha_{r}}+\bar{C}_{\alpha_{1}} \dot{P}^{\alpha_{1}}-H_{\Psi}\right) .
\end{aligned}
$$

This action accounts for unfree gauge symmetry in two ways. First, the nonminimal sector is asymmetric with the minimal one unlike the usual BFV formalism. Second, the secondary constraints, being a part of the BRST generator $Q$, may be explicitly time dependent, even though the original action does not involve time explicitly. Both of 
these features do not obstruct the usual reasoning that justifies $\Psi$ independence of the transition amplitude for this action,

$$
Z_{\Psi}=\int[D \Phi] \exp \left\{\frac{i}{\hbar} S_{\mathrm{BRST}}^{\Psi}\right\}
$$

where $\Phi=\left\{q^{i}, p_{i}, \lambda^{\alpha_{1}}, \pi_{\alpha_{1}}, C^{\alpha_{1}}, \bar{P}_{\alpha_{1}}, C^{\alpha_{2}}, \bar{P}_{\alpha_{2}}, \ldots, C^{\alpha_{n}}, \bar{P}_{\alpha_{n}}\right.$, $\left.P^{\alpha_{1}}, \bar{C}_{\alpha_{1}}\right\}$.

Let us consider a theory (19) with constraints (22), (24), and (25), with the involution relations

$$
\left\{\stackrel{(r)}{T}_{\alpha_{r}}(q, p), \stackrel{(s)}{T} \alpha_{s}(q, p)\right\}=U_{\alpha_{r}}^{\gamma_{t} \alpha_{s}}(q, p) \stackrel{(t)}{T} \gamma_{t}(q, p),
$$

where $r, s, t=1, \ldots, n$. Assume that BRST generator $Q_{\text {min }}$ and Hamiltonian $\mathcal{H}$ are at most linear in the ghost momenta,

$$
\begin{aligned}
Q_{\min } & =\sum_{r=1}^{n} C^{\alpha_{r}} \stackrel{(r)}{T}_{\alpha_{r}}+\frac{1}{2} \sum_{r, s, t=1}^{n} C^{\beta_{s}} C^{\alpha_{r}} U_{\alpha_{r} \beta_{s}}^{\gamma_{t}} \bar{P}_{\gamma_{t}}, \\
\mathcal{H}= & H(q, p)+\sum_{r=1}^{n} C^{\alpha_{r}}\left(\sum_{s=1}^{n} \stackrel{(r)}{V}_{\alpha_{r}}^{\beta_{s}}(q, p, \lambda) \bar{P}_{\beta_{s}}\right. \\
& \left.+\stackrel{(r)}{\Gamma}{ }_{\alpha_{r}}^{\beta_{r-1}}(q, p, \lambda) \bar{P}_{\beta_{r-1}}\right) .
\end{aligned}
$$

We also assume the following form of gauge-fixed Hamiltonian:

$$
\begin{aligned}
H_{\Psi}= & \mathcal{H}+\{Q, \Psi\}=\mathcal{H}+\lambda^{\alpha_{1}} \stackrel{(1)}{T}_{\alpha_{1}}+\pi_{\alpha_{1}} \chi^{\alpha_{1}}+\bar{P}_{\alpha_{1}} P^{\alpha_{1}} \\
& +\sum_{r=1}^{n} \bar{C}_{\alpha_{1}}\left\{\chi^{\alpha_{1}}, \stackrel{(r)}{T}_{\alpha_{r}}\right\} C^{\alpha_{r}} .
\end{aligned}
$$

This is automatically true if the gauge conditions $\chi$ Poisson-commute to structure functions $U$ in the involution relations (54). Given the action, path integral (53) reads

$$
\begin{aligned}
Z_{\Psi}= & \int[D \Phi] \exp \left\{\frac { i } { \hbar } \int d t \left[p_{i} \dot{q}^{i}-H(q, p)-\lambda^{\alpha_{1}} \stackrel{(1)}{T}_{\alpha_{1}}\right.\right. \\
& +\pi_{\alpha_{1}}\left(\dot{\lambda}^{\alpha_{1}}-\chi^{\alpha_{1}}\right)-\sum_{r=1}^{n} \bar{C}_{\alpha_{1}}\left\{\chi^{\alpha_{1}}, \stackrel{(r)}{T}_{\alpha_{r}}\right\} C^{\alpha_{r}} \\
& +\bar{P}_{\alpha_{1}}\left(\dot{C}^{\alpha_{1}}+\sum_{s=1}^{n} \stackrel{(s)}{V}_{\beta_{s}}^{\alpha_{1}}(q, p, \lambda) C^{\beta_{s}}\right) \\
& +P^{\alpha_{1}}\left(\bar{P}_{\alpha_{1}}+\dot{\bar{C}}_{\alpha_{1}}\right)+\sum_{k=2}^{n} \bar{P}_{\alpha_{k}}\left(\left(\delta_{\beta_{k}}^{\alpha_{k}} \frac{d}{d t}+\stackrel{(k)}{V} \underset{\beta_{k}}{\alpha_{k}}\right) C^{\beta_{k}}\right. \\
& \left.\left.\left.+\sum_{m=k+1}^{n} \stackrel{(m)}{V}{ }_{\beta_{k}}^{\alpha_{k}} C^{\beta_{m}}+\stackrel{(k-1)}{\Gamma}{ }_{\beta_{k}} C^{\beta_{k-1}}\right)\right]\right\}
\end{aligned}
$$

where $\quad \Phi=\left\{q^{i}, p_{i}, \lambda^{\alpha_{1}}, \pi_{\alpha_{1}}, C^{\alpha_{1}}, \bar{P}_{\alpha_{1}}, C^{\alpha_{2}}, \bar{P}_{\alpha_{2}}, \ldots, C^{\alpha_{n}}\right.$, $\left.\bar{P}_{\alpha_{n}}, P^{\alpha_{1}}, \bar{C}_{\alpha_{1}}\right\}$. Integrating in path integral (58) over $P^{\alpha_{1}}$, $\bar{P}_{\alpha_{1}}$, we arrive at the following answer for the transition amplitude:

$$
\begin{aligned}
& Z_{\Psi}=\int\left[D \Phi^{\prime}\right] \exp \left\{\frac { i } { \hbar } \int d t \left[p_{i} \dot{q}^{i}-H(q, p)-\lambda^{\alpha_{1}}{ }_{\alpha_{1}}^{(1)}\right.\right. \\
& +\pi_{\alpha_{1}}\left(\dot{\lambda}^{\alpha_{1}}-\chi^{\alpha_{1}}\right) \sum_{r=1}^{n} \bar{C}_{\alpha_{1}}\left\{\chi^{\alpha_{1}}, \stackrel{(r)}{T}{ }_{\alpha_{r}}\right\} C^{\alpha_{r}} \\
& -\bar{C}_{\alpha_{1}}\left(\dot{C}^{\alpha_{1}}+\sum_{s=1}^{n} \stackrel{(s)}{V}_{\beta_{s}}^{\alpha_{1}}(q, p, \lambda) C^{\beta_{s}}\right) \\
& +\sum_{k=2}^{n} \bar{P}_{\alpha_{k}}\left(\left(\delta_{\beta_{k}}^{\alpha_{k}} \frac{d}{d t}+\stackrel{(k)}{V}_{\beta_{k}}^{\alpha_{k}}\right) C^{\beta_{k}}\right. \\
& \left.\left.\left.+\sum_{m=k+1}^{n} \stackrel{(m)}{V}{ }_{\beta_{m}}^{\alpha_{k}} C^{\beta_{m}}+\stackrel{(k-1)}{\Gamma}{ }_{\beta_{k-1}} C^{\beta_{k-1}}\right)\right]\right\},
\end{aligned}
$$

where $\quad \Phi^{\prime}=\left\{q^{i}, p_{i}, \lambda^{\alpha_{1}}, \pi_{\alpha_{1}}, C^{\alpha_{1}}, C^{\alpha_{2}}, \bar{P}_{\alpha_{2}}, \ldots, C^{\alpha_{n}}, \bar{P}_{\alpha_{n}}\right.$, $\left.\bar{C}_{\alpha_{1}}\right\}$.

Let us discuss the path integral (59). The first line in (59) is the original action (19) and the gauge-fixing term. The second line is the FP term for the gauge transformations (31), (32). The third line has a natural interpretation from the viewpoint of the modified FP ansatz in Lagrangian formalism (11), (13). The ghost momenta $\bar{P}_{\alpha_{k}}, k=2, \ldots, n$ can be viewed as Fourier multipliers at the constraints imposed on ghosts,

$$
\begin{aligned}
& \left(\delta_{\beta_{k}}^{\alpha_{k}} \frac{d}{d t}+\stackrel{(k)}{V}{ }_{\beta_{k}}^{\alpha_{k}}(q, p, \lambda)\right) C^{\beta_{k}}+\sum_{m=k+1}^{n} \stackrel{(m)}{V}{ }_{\beta_{m}}^{\alpha_{k}}(q, p, \lambda) C^{\beta_{m}} \\
& \quad+\stackrel{(k-1)}{\Gamma}{ }_{\beta_{k-1}}^{\alpha_{l}}(q, p, \lambda) C^{\beta_{k-1}}=0
\end{aligned}
$$

These ghost constraints mirror the equations imposed on gauge parameters in Hamiltonian formalism (33), (34). So, Eq. (60) represents Hamiltonian form of the constraints (8) imposed on the ghosts in the case of unfree gauge symmetry. With this regard, the path integral (59) represents the modified FP recipe (11), (13) for the Hamiltonian action (19), gauge symmetry (31), (32), and the constraints (33), (34) on the gauge parameters. So, proceeding from the amplitude (53) in the general Hamiltonian BFV-BRST formalism for unfree gauge symmetry, in the case without higher order ghost contributions (55), (57), we arrive at the modified FP path integral (11), (13). 


\section{EXAMPLE: TRACELESS MASSLESS SPIN $s$ GAUGE FIELDS}

\section{A. Lagrangian, completion functions, and unfree gauge symmetry}

Let us consider a theory of traceless symmetric tensor field $\varphi_{\mu_{1} \ldots \mu_{s}}, \varphi^{\nu}{ }_{\nu \mu_{3} \ldots \mu_{s}}=0$, in $d$-dimensional Minkowski space. The metric is chosen mostly negative, $\eta_{\mu \nu}=$ $\operatorname{diag}(1,-1, \ldots,-1)$. The Lagrangian reads [3]

$$
\begin{aligned}
\mathcal{L}= & (-1)^{s}\left(\frac{1}{2} \partial_{\nu} \varphi_{\mu_{1} \ldots \mu_{s}} \partial^{\nu} \varphi^{\mu_{1} \ldots \mu_{s}}\right. \\
& \left.-\frac{s}{2} \partial^{\nu} \varphi_{\nu \mu_{2} \ldots \mu_{s}} \partial_{\rho} \varphi^{\rho \mu_{2} \ldots \mu_{s}}\right) \\
& +(-1)^{s} \frac{s}{2} \partial^{\nu}\left(\varphi_{\nu \mu_{2} \ldots \mu_{s}} \partial_{\rho} \varphi^{\rho \mu_{2} \ldots \mu_{s}}\right) .
\end{aligned}
$$

The last term is a total divergence, so it does not contribute to EoMs. We include it for convenience when constructing the Hamiltonian formalism.

The above Lagrangian describes irreducible massless spin- $s$ representation of Poincaré group. One of the advantages of this form of the irreducible higher spin theory, comparing to the Frondsdal Lagrangian [17], is that it does not involve auxiliary fields. This Lagrangian can be viewed as higher spin extension of linearized UG [1,2]. In this section, we utilize this model for exemplifying all the generalities about unfree gauge symmetry considered above in this paper.

The field equations for the Lagrangian (61) read

$$
\begin{aligned}
\frac{\delta S}{\delta \varphi^{\mu_{1} \ldots \mu_{s}}} \equiv & -(-1)^{s}\left[\square \varphi_{\mu_{1} \ldots \mu_{s}}-s \partial_{\left(\mu_{1}\right.} \partial^{\nu} \varphi_{\left.\nu \mu_{2} \ldots \mu_{s}\right)}\right. \\
& \left.+\frac{s(s-1)}{d+2 s-4} \eta_{\left(\mu_{1} \mu_{2}\right.} \partial^{\nu} \partial^{\rho} \varphi_{\left.\nu \rho \mu_{3} \ldots \mu_{s}\right)}\right]=0,
\end{aligned}
$$

where round brackets $\left(\mu_{1} \ldots \mu_{s}\right)$ mean symmetrization of all the included indices. Taking the divergence of the lhs, we get the differential consequence, cf. (15),

$$
\begin{aligned}
\partial^{\mu_{1}} \frac{\delta S}{\delta \varphi^{\mu_{1} \ldots \mu_{s}}} \equiv & (-1)^{s-1} \frac{d+2 s-6}{d+2 s-4}\left(\partial_{\left(\mu_{1}\right.} \tau_{\left.\mu_{2} \ldots \mu_{s-1}\right)}\right) \\
& \left.-\frac{2}{d+2 s-6} \eta_{\left(\mu_{1} \mu_{2}\right.} \partial^{\lambda} \tau_{\left.\lambda \ldots \mu_{s-2}\right)}\right) \approx 0,
\end{aligned}
$$

where $\tau_{\mu_{2} \ldots \mu_{s-1}}$ is a double divergence of the field,

$$
\tau_{\mu_{1} \ldots \mu_{s-2}}=\partial^{\rho} \partial^{\nu} \varphi_{\nu \rho \ldots \mu_{s-2}} .
$$

Relation (63) means that $\tau$ reduces on-shell to the element of the kernel of first-order differential operator. For $s=2, \tau$ is a scalar, and relation (63) means just $\partial_{\mu} \tau=0$, so $\tau$ is just on-shell constant. In this case, the kernel is onedimensional. For $s \geq 3$, relation (63) means

$$
\tau_{\mu_{1} \ldots \mu_{s-2}} \approx \Lambda_{\mu_{1} \ldots \mu_{s-2}}
$$

with $\Lambda_{\mu_{1} \ldots, \mu_{s-2}}$ being a solution of conformal Killing tensor equations,

$$
\partial_{\left(\mu_{1}\right.} \Lambda_{\left.\mu_{2} \ldots \mu_{s-1}\right)}-\frac{2}{d+2 s-6} \eta_{\left(\mu_{1} \mu_{2}\right.} \partial^{\nu} \Lambda_{\left.\nu \ldots \mu_{s-2}\right)}=0 .
$$

The space of conformal Killing tensors is finite dimensional, so $\tau$ is a completion function. Specific $\Lambda$ is defined by the asymptotic behavior of the fields. For example, if $\varphi$ vanishes at infinity, then $\Lambda=0$. In this most simple case, $\tau$ still remains a nontrivial completion function as it depends on derivatives of the fields off-shell. This linear function of $\partial^{2} \varphi$ vanishes on-shell, while it is not a linear combination of the Lagrangian equations (62). We detail the case of nonvanishing $\Lambda$ below for $s=3$.

Once $\tau$ (64) is a completion function, relation (63) should be understood as modified Noether identity (3) because it binds Lagrangian equations with completion functions,

$$
\begin{gathered}
\partial^{\mu_{1}} \frac{\delta S}{\delta \varphi^{\mu_{1} \ldots \mu_{s}}}+(-1)^{s} \frac{d+2 s-6}{d+2 s-4}\left(\partial_{\left(\mu_{1}\right.} \tau_{\left.\mu_{2} \ldots \mu_{s-1}\right)}\right. \\
\left.-\frac{2}{d+2 s-6} \eta_{\left(\mu_{1} \mu_{2}\right.} \partial^{\lambda} \tau_{\left.\lambda \ldots \mu_{s-2}\right)}\right) \equiv 0 .
\end{gathered}
$$

Given the identities (3), it defines unfree gauge symmetry of the action: the coefficients at EoMs define the gauge generators (5), while the ones at completion functions define the equations (6) constraining the gauge parameters. In this way, the identities (67) define unfree gauge symmetry

$$
\delta_{\varepsilon} \varphi_{\mu_{1} \ldots \mu_{s}}=s \partial_{\left(\mu_{1}\right.} \varepsilon_{\left.\mu_{2} \ldots \mu_{s}\right)},
$$

where $\varepsilon_{\mu_{1} \ldots \mu_{s-1}}$ are traceless symmetric gauge parameters, $\varepsilon^{\nu}{ }_{\nu \mu_{2} \ldots \mu_{s-1}}=0$, subject to the transversality conditions

$$
\partial^{\nu} \varepsilon_{\nu \mu_{2} \ldots \mu_{s-1}}=0 .
$$

Transformations (68) and constraints (69) are noticed in the paper [3] where the Lagrangian (61) is proposed. The completion functions (64), (65) are noticed here for the first time.

\section{B. Covariant degree of freedom count}

Let us now apply formula (18) to verify d.o.f number of the spin-s theory (61) in explicitly covariant way. Given the EoMs (62), symmetry transformations (68), gauge identities (67), and constraints on gauge parameters (69), we can compute all the ingredients needed to count the d.o.f number by the recipe (18). The number of the second-order $\left(o_{e}=2\right)$ Lagrangian equations (62) corresponds to the number of independent components of traceless $s$-rank tensor, 


$$
\begin{aligned}
n_{e} & =\left(\begin{array}{c}
d+s-1 \\
s
\end{array}\right)-\left(\begin{array}{c}
d+s-3 \\
s-2
\end{array}\right) \\
& =\frac{(d+s-3) !}{s !(d-1) !}\left(d^{2}+d(2 s-3)-2(s-1)\right) .
\end{aligned}
$$

The number of first-order $\left(o_{s}=1\right)$ symmetry transformations (68) and third-order $\left(o_{i}=1+2=3\right)$ gauge identities (67) equals the number of independent components of traceless $(s-1)$-tensor,

$$
\begin{aligned}
n_{s}=n_{i} & =\left(\begin{array}{c}
d+s-1 \\
s-1
\end{array}\right)-\left(\begin{array}{c}
d+s-4 \\
s-3
\end{array}\right) \\
& =\frac{(d+s-4) !}{(s-1) !(d-1) !}\left(d^{2}+d(2 s-5)-2(s-2)\right) .
\end{aligned}
$$

There exist second-order $\left(o_{c}=1+1=2\right)$ constraints on gauge parameters (69), whose number coincides with the number of independent components of traceless $(s-2)$ tensor,

$$
\begin{aligned}
n_{c} & =\left(\begin{array}{c}
d+s-3 \\
s-2
\end{array}\right)-\left(\begin{array}{c}
d+s-5 \\
s-4
\end{array}\right) \\
& =\frac{(d+s-5) !}{(s-2) !(d-1) !}\left(d^{2}+d(2 s-7)-2(s-3)\right) .
\end{aligned}
$$

So, the expression (18) for d.o.f counting in case of a theory (61) reads

$$
\begin{aligned}
N_{\text {d.o.f }}= & {\left[\left(\begin{array}{c}
d+s-1 \\
s
\end{array}\right)-\left(\begin{array}{c}
d+s-3 \\
s-2
\end{array}\right)\right] \cdot 2 } \\
& -\left[\left(\begin{array}{c}
d+s-1 \\
s-1
\end{array}\right)-\left(\begin{array}{c}
d+s-4 \\
s-3
\end{array}\right)\right] \cdot 1 \\
& -\left[\left(\begin{array}{c}
d+s-1 \\
s-1
\end{array}\right)-\left(\begin{array}{c}
d+s-4 \\
s-3
\end{array}\right)\right] \cdot 3 \\
& +\left[\left(\begin{array}{c}
d+s-3 \\
s-2
\end{array}\right)-\left(\begin{array}{c}
d+s-5 \\
s-4
\end{array}\right)\right] \cdot 2 .
\end{aligned}
$$

For $d=4$, this means

$$
\left.N_{\text {d.o.f }}\right|_{d=4}=(s+1)^{2} \cdot 2-s^{2} \cdot 1-s^{2} \cdot 3+(s-1)^{2} \cdot 2=4 \text {. }
$$

Four d.o.f by the phase-space count corresponds to two Lagrangian modes, which is correct number for massless spin- $s$ field in $d=4$.

\section{Completion functions, asymptotics, moduli space for $s=3$}

Let us elaborate on the contribution of field asymptotics to the completion functions in the simplest higher spin case. For $s=3$, Lagrangian (61) and field equations (62) read

$$
\begin{gathered}
\mathcal{L}=-\left(\frac{1}{2} \partial_{\lambda} \varphi_{\mu \nu \rho} \partial^{\lambda} \varphi^{\mu \nu \rho}-\frac{3}{2} \partial^{\mu} \varphi_{\mu \nu \rho} \partial_{\lambda} \varphi^{\lambda \nu \rho}\right) \\
-\frac{3}{2} \partial^{\mu}\left(\varphi_{\mu \nu \rho} \partial_{\lambda} \varphi^{\lambda \nu \rho}\right), \quad \varphi^{\nu}{ }_{\nu \mu}=0, \\
\frac{\delta S}{\delta \varphi^{\mu \nu \rho}} \equiv \square \varphi_{\mu \nu \rho}-3 \partial_{(\mu} \partial^{\lambda} \varphi_{\lambda \nu \rho)} \\
+\frac{6}{d+2} \eta_{(\mu \nu} \partial^{\lambda} \partial^{\sigma} \varphi_{\sigma \lambda \rho)}=0 .
\end{gathered}
$$

Taking the divergence of the field equations, we get the differential consequence

$$
\begin{aligned}
\partial^{\lambda} \frac{\delta S}{\delta \varphi^{\lambda \mu \nu}} \equiv & \frac{2 d}{d+2}\left(\partial_{(\mu} \partial^{\rho} \partial^{\lambda} \varphi_{\lambda \rho \nu)}\right. \\
& \left.-\frac{1}{d} \eta_{\mu_{2} \mu_{3}} \partial^{\lambda} \partial^{\rho} \partial^{\nu} \varphi_{\nu \rho \lambda}\right) \approx 0 .
\end{aligned}
$$

Introduce the notation

$$
\tau_{\mu}=\partial^{\nu} \partial^{\lambda} \varphi_{\mu \nu \lambda} .
$$

Relation (77) means that $\tau_{\mu}$ must obey on-shell the equation for conformal Killing vector field,

$$
\partial_{\mu} \tau_{\nu}+\partial_{\nu} \tau_{\mu}-\frac{2}{d} \eta_{\mu \nu} \partial^{\rho} \tau_{\rho} \approx 0
$$

The general solution of the conformal Killing equation reads

$$
\begin{aligned}
\partial_{\mu} \Lambda_{\nu}+\partial_{\nu} \Lambda_{\mu}-\frac{2}{d} \eta_{\mu \nu} \partial_{\rho} \Lambda^{\rho}= & 0 \Leftrightarrow \Lambda_{\mu}=a_{\mu}+2 \eta_{\mu \nu} \omega^{\nu \rho} x_{\rho} \\
& +\lambda x_{\mu}+b^{\nu}\left(2 x_{\mu} x_{\nu}-\eta_{\mu \nu} x_{\rho} x^{\rho}\right),
\end{aligned}
$$

where $a_{\mu}, \lambda, b^{\mu}, \omega^{\mu \nu}=-\omega^{\nu \mu}$ are arbitrary (integration) constants, so there are $\frac{(d+2)(d+1)}{2}$ constant parameters. Relation (79) means that $\tau_{\mu}$ reduces on-shell to Killing vector (80),

$$
\partial_{\nu} \partial_{\lambda} \varphi^{\mu \nu \lambda} \approx \Lambda^{\mu}(x ; \lambda, a, b, \omega)
$$

Let us shift the notation (78): $\tau_{\mu}=\partial^{\nu} \partial^{\lambda} \varphi_{\mu \nu \lambda}-\Lambda_{\mu}(x ; \lambda$, $a, b, \omega)$. Then, $\tau_{\mu}$ vanishes on-shell,

$$
\tau_{\mu} \equiv \partial^{\nu} \partial^{\lambda} \varphi_{\mu \nu \lambda}-\Lambda_{\mu}(x ; \lambda, a, b, \omega) \approx 0 .
$$

So, we have a function of the field derivatives such that vanishes on-shell, while it is not a linear combination of the lhs of Lagrangian equations (76) and their derivatives. This means that $\tau_{\mu}$ is a completion function, according to definition (1). Relation (82) can be considered as spin-3 
analogue of relation $\tau \equiv R-\Lambda \approx 0$ in UG. There are two distinctions, however. First, in the case of UG, we have one completion function which involves one constant parameter. In the case of spin-3, we have $d$ completion functions involving $\frac{(d+1)(d+2)}{2}$ constant parameters. Second, in the case of UG, the completion function does not depend on space-time coordinates, while for $s=3$ there is explicit $x$ dependence. We see that the number of modular parameters does not directly correlate to the number of completion functions. Also, completion functions can be explicitly $x$ dependent, even if the Lagrangian is translation invariant. Specific modular parameters $\lambda, a, b, \omega$ are defined by asymptotics of the fields. If the fields tend to zero at infinity, all the parameters vanish, while the equation $\tau_{\mu}=0$ will remain a nontrivial relation anyway, not a differential consequence of Lagrangian equations.

Notice that field equations (76) admit the solutions such that compatible with any modular parameters $\lambda, a, b, \omega$ in the completion function (82). Let $\varkappa_{\mu \nu \rho}^{(0)}$ be a general solution vanishing at infinity. It includes the Cauchy data, corresponding to four local physical d.o.f in $4 d$ case. Double divergence of $\varkappa_{\mu \nu \rho}^{(0)}$ inevitably vanishes. There is another solution, $\varkappa_{\mu \nu \rho}$, with different asymptotics which includes the same number of local Cauchy data and arbitrary modular parameters,

$$
\begin{aligned}
\varkappa_{\mu \nu \rho}= & \varkappa_{\mu \nu \rho}^{(0)}+A\left[a_{(\mu} x_{\nu} x_{\rho)}-\frac{1}{d+2} \eta_{(\mu \nu} a_{\rho)} x_{\lambda} x^{\lambda}\right. \\
& \left.-\frac{2}{d+2} \eta_{(\mu \nu} x_{\rho)} a_{\lambda} x^{\lambda}\right]+B\left[\eta_{(\mu \alpha} \omega^{\alpha \beta} x_{\beta} x_{\nu} x_{\rho)}\right. \\
& \left.-\frac{1}{d+2} \eta_{(\mu \nu} \eta_{\rho) \alpha} \omega^{\alpha \beta} x_{\beta} x_{\lambda} x^{\lambda}\right]+C \lambda\left[x_{(\mu} x_{\nu} x_{\rho)}\right. \\
& \left.-\frac{3}{d+2} \eta_{(\mu \nu} x_{\rho)} x_{\lambda} x^{\lambda}\right]+D b^{\lambda} x_{\lambda} x_{(\mu} x_{\nu} x_{\rho)} \\
& +E b_{(\mu} x_{\nu} x_{\rho)} x_{\lambda} x^{\lambda}+F \eta_{(\mu \nu} b_{\rho)} x_{\lambda} x^{\lambda} x_{\sigma} x^{\sigma} \\
& +G \eta_{(\mu \nu} x_{\rho)} b^{\lambda} x_{\lambda} x_{\sigma} x^{\sigma},
\end{aligned}
$$

where

$$
\begin{aligned}
& A=\frac{3}{(d+4)(d-1)}, \quad B=\frac{6}{(d+4)(d+1)}, \\
& C=\frac{1}{(d+4)(d-1)}, \quad D=\frac{2\left(d^{2}+7 d-6\right)}{(d+6)(d+4)\left(d^{2}-1\right)}, \\
& E=-\frac{3\left(d^{2}+3 d-6\right)}{(d+6)(d+4)\left(d^{2}-1\right)}, \\
& F=\frac{3\left(d^{2}+3 d-6\right)}{(d+6)(d+4)(d+2)\left(d^{2}-1\right)}, \\
& G=-\frac{24 d}{(d+6)(d+4)(d+2)\left(d^{2}-1\right)} .
\end{aligned}
$$

For the solution $\varkappa$, the double divergence of the field is a general conformal Killing vector (80),

$$
\partial^{\nu} \partial^{\lambda} \varkappa_{\mu \nu \lambda}=\Lambda_{\mu}(x ; \lambda, a, b, \omega)
$$

Once we have the completion function $\tau_{\mu}$ (82), relation (77) can be reformulated as modified Noether identity (3),

$\partial^{\lambda} \frac{\delta S}{\delta \varphi^{\lambda \mu \nu}}-\frac{d}{d+2}\left(\partial_{\mu} \tau_{\nu}+\partial_{\nu} \tau_{\mu}-\frac{2}{d} \eta_{\mu \nu} \partial_{\lambda} \tau^{\lambda}\right) \equiv 0$.

Given the modified Noether identity, the coefficient at the equations defines unfree gauge variation of the field, while the coefficient at completion function defines the equation constraining the gauge parameters. In this way, we get unfree gauge symmetry of Lagrangian (75),

$$
\begin{gathered}
\delta_{\varepsilon} \varphi_{\mu \nu \lambda}=\partial_{\mu} \varepsilon_{\nu \lambda}+\partial_{\nu} \varepsilon_{\lambda \mu}+\partial_{\lambda} \varepsilon_{\mu \nu}, \\
\partial^{\nu} \varepsilon_{\nu \mu}=0,
\end{gathered}
$$

where the gauge parameters are symmetric traceless tensors $\varepsilon_{\mu \nu}=\varepsilon_{\nu \mu}, \varepsilon^{\nu}{ }_{\nu}=0$.

\section{Constrained Hamiltonian formalism for $s=3$ case}

Hamiltonian formalism for the theory (61) is worked out in the paper [3]. Our analysis extends the consideration of [3] in two respects. First, the paper [3] assumed that fields vanish at infinity. We admit nontrivial boundary conditions for the fields, and reveal contribution of the modular parameters to the Hamiltonian constraints. Second, we demonstrate that involution relations of constraints and Hamiltonian define the unfree gauge symmetry.

We begin constructing the Hamiltonian formalism with $1+(d-1)$ decomposition of the fields such that accounts for the traceless condition. The indices $\mu, \nu, \ldots=$ $0,1, \ldots, d-1$ are split into 0 and $i, j, \ldots=1, \ldots, d-1$. Metrics $\eta_{i j}=\operatorname{diag}(-1, \ldots,-1)$. Introduce abbreviation

$$
\varphi_{0 i j} \equiv \tilde{\varphi}_{i j}+\frac{1}{d-1} \eta_{i j} \varphi, \quad \eta^{i j} \tilde{\varphi}_{i j}=0
$$

and notice the consequences of symmetry and traceless properties of $\varphi_{\mu \nu \lambda}$,

$$
\varphi^{0}{ }_{00}=-\varphi^{i}{ }_{i 0} \equiv-\varphi, \quad \varphi^{0}{ }_{0 i}=-\varphi_{j i}^{j},
$$

where $\varphi^{j}{ }_{j i}=\eta^{j k} \varphi_{k j i}$. Given relations (89), (90), Lagrangian (75), being expressed in terms of the variables $\varphi_{i j k}, \tilde{\varphi}_{i j}, \varphi$, modulo total time derivative reads 


$$
\begin{aligned}
\mathcal{L}= & -\frac{1}{2} \dot{\varphi}_{i j k} \dot{\varphi}^{i j k}+\frac{3}{2} \dot{\varphi}_{i j}{ }^{j} \dot{\varphi}^{i k}{ }_{k}+\dot{\varphi}^{2}+3 \dot{\varphi}_{i j k} \partial^{k} \tilde{\varphi}^{j i} \\
& -6 \dot{\varphi}_{i j}{ }^{j} \partial_{k} \tilde{\varphi}^{k i}-\frac{3}{d-1} \dot{\varphi}_{i j}{ }^{j} \partial^{i} \varphi+3 \dot{\varphi} \partial_{i} \varphi^{i j}{ }_{j} \\
& -\frac{1}{2}\left(\partial_{i} \varphi_{j k l} \partial^{i} \varphi^{j k l}+3 \partial_{i} \varphi_{j k}{ }^{k} \partial^{i} \varphi^{j l}{ }_{l}+3 \partial_{i} \tilde{\varphi}_{j k} \partial^{i} \tilde{\varphi}^{j k}\right. \\
& \left.+\frac{d^{2}+d-8}{(d-1)^{2}} \partial_{i} \varphi \partial^{i} \varphi\right)+\frac{3}{2}\left(\partial^{i} \varphi_{i k l} \partial_{j} \varphi^{j k l}+\partial^{i} \varphi_{i k}{ }^{k} \partial_{j} \varphi^{j l}{ }_{l}\right. \\
& \left.+2 \partial^{i} \tilde{\varphi}_{i k} \partial_{j} \tilde{\varphi}^{j k}+\frac{4}{d-1} \partial^{i} \tilde{\varphi}_{i j} \partial^{j} \varphi\right) .
\end{aligned}
$$

The Lagrangian does not include $\dot{\tilde{\varphi}}_{i j}$. Making the Legendre transform with respect to $\dot{\varphi}_{i j k}$ and $\dot{\varphi}$, the action is brought to the Hamiltonian form

$$
S_{H}=\int d^{d} x\left(\Pi^{i j k} \dot{\varphi}_{i j k}+\Pi \dot{\varphi}-H-\tilde{\varphi}^{i j} \tilde{T}_{i j}\right)
$$

where the Hamiltonian reads

$$
\begin{aligned}
H= & -\frac{1}{2} \Pi^{i j k} \Pi_{i j k}+\frac{3}{2} \frac{1}{d} \Pi^{i j}{ }_{j} \Pi_{i k}{ }^{k}+\frac{1}{4} \Pi^{2} \\
& +\frac{3}{d(d-1)} \Pi^{i j} \partial_{j} \varphi-\frac{3}{2} \Pi \partial_{i} \varphi^{i j}{ }_{j} \\
& +\frac{1}{2}\left(\partial_{i} \varphi_{j k l} \partial^{i} \varphi^{j k l}+3 \partial_{i} \varphi_{j k}{ }^{k} \partial^{i} \varphi^{j l}{ }_{l}+\frac{d+3}{d} \partial_{i} \varphi \partial^{i} \varphi\right) \\
& -\frac{3}{2}\left(\partial^{i} \varphi_{i k l} \partial_{j} \varphi^{j k l}-\frac{1}{2} \partial^{i} \varphi_{i k}{ }^{k} \partial_{j} \varphi^{j l}{ }_{l}\right)
\end{aligned}
$$

and

$$
\tilde{T}_{i j} \equiv-3\left(\partial^{k} \Pi_{k i j}-\frac{1}{d-1} \eta_{i j} \partial_{k} \Pi^{k l}{ }_{l}\right)=0
$$

$\eta^{i j} \tilde{T}_{i j} \equiv 0$, are the primary constraints, with $\tilde{\varphi}^{i j}$ being Lagrange multipliers.

Let us examine stability of primary constraints (94),

$$
\begin{aligned}
\dot{\tilde{T}}_{i j} & =\left\{\tilde{T}_{i j}, H_{0}\right\} \\
& =-\left(\delta_{(i}^{k} \partial_{j)}-\frac{1}{d-1} \eta_{i j} \partial^{k}\right) T^{\prime}{ }_{k}=0,
\end{aligned}
$$

where

$T^{\prime}{ }_{i}=-3\left(\partial_{i} \Pi-\partial_{i} \partial^{j} \varphi_{j k}{ }^{k}-2 \Delta \varphi_{i j}{ }^{j}+2 \partial^{j} \partial^{k} \varphi_{k j i}\right)$.

The coefficient at $T^{\prime}{ }_{i}$ in relation (95) is a linear differential operator with the finite kernel. The equation for the null vectors of the operator reads

$$
\partial_{i} \Lambda_{j}+\partial_{j} \Lambda_{i}-\frac{1}{d-1} \eta_{i j} \partial_{k} \Lambda^{k}=0
$$

The equation above defines the conformal Killing vector field in $(d-1)$-dimensional space. The space of conformal Killing vectors is finite dimensional. There is a subtlety, however. It concerns the fact that the parameters defining the solution to Eq. (97) may be time dependent. This can be understood from the fact that solution of (97) should explicitly depend on space coordinates $x^{i}$, while the theory is Lorentz invariant. Then, Lorentz boost will inevitably bring time dependence to any solution of (97). The time dependence is fixed, as we shall see below, by further stability conditions. Stability condition (95) means that $T^{\prime}{ }_{i}$ (96) reduce to the solution of Eq. (97), i.e., we arrive at secondary constraints,

$$
T_{i} \equiv T^{\prime}{ }_{i}-\Lambda_{i}(x)=0
$$

Given the secondary constraints, they have to conserve. The conservation condition reads

$$
\begin{aligned}
\dot{T}_{i}= & \partial_{0} T_{i}+\left\{T_{i}, H\right\}=-\partial_{0} \Lambda_{i}(x) \\
& -2\left(\delta_{i}^{(j} \partial^{k)}-\frac{1}{d-1} \eta^{j k} \partial_{i}\right) \tilde{T}_{j k}+\partial_{i} T=0 .
\end{aligned}
$$

Relation (99) means we have tertiary constraint

$$
T \equiv T^{\prime}+\Lambda_{0}(x)=0
$$

where

$$
T^{\prime}=-3\left(\frac{1}{d-1} \partial^{i} \Pi_{i j}^{j}+\Delta \varphi\right)
$$

and $\Lambda_{0}(x)$ is connected with $\Lambda_{i}(x)$ of (98) by the relation

$$
\partial_{0} \Lambda_{i}+\partial_{i} \Lambda_{0}=0
$$

Given tertiary constraint (100), it has to conserve,

$$
\begin{aligned}
\dot{T}= & \partial_{0} T+\{T, H\}=\partial_{0} \Lambda_{0} \\
& -\frac{1}{d-1} \partial^{i} T_{i}-\frac{1}{d-1} \partial^{i} \Lambda_{i}=0 .
\end{aligned}
$$

This relation does not result in any new constraint, while it is consistent if $\Lambda_{0}(x)$ and $\Lambda_{i}(x)$ are connected by one more relation

$$
\partial_{0} \Lambda_{0}-\frac{1}{d-1} \partial^{i} \Lambda_{i}=0
$$

Relations (97), (102), and (104) taken together are just $1+$ $(d-1)$ decomposition of conformal Killing equation (80) in $d$ dimensions. So, $\Lambda_{i}(x), \Lambda_{0}(x)$ are the components of conformal Killing vector, 


$$
\begin{aligned}
\Lambda_{i}= & a_{i}+2\left(\omega_{i 0} x^{0}+\omega_{i j} x^{j}\right)+\lambda x_{i} \\
& +2\left(b_{0} x^{0}+b_{j} x^{j}\right) x_{i}-b_{i}\left(x_{0} x^{0}+x_{j} x^{j}\right) \\
\Lambda_{0}=a_{0} & +2 w_{0 i} x^{i}+\lambda x_{0}+b_{0}\left(x_{0} x^{0}-x_{i} x^{i}\right)+2 b_{i} x^{i} x_{0} .
\end{aligned}
$$

As soon as the Dirac-Bergrmann algorithm is completed, let us summarize its results. Complete set of constraints reads

$$
\begin{aligned}
\tilde{T}_{i j} & =-3\left(\partial^{k} \Pi_{k i j}-\frac{1}{d-1} \eta_{i j} \partial_{k} \Pi_{l}^{k l}\right), \\
T_{i} & =-3\left(\partial_{i} \Pi-\partial_{i} \partial^{j} \varphi_{j k}{ }^{k}-2 \Delta \varphi_{i j}{ }^{j}+2 \partial^{j} \partial^{k} \varphi_{k j i}\right)-\Lambda_{i}, \\
T & =-3\left(\frac{1}{d-1} \partial^{i} \Pi_{i j}^{j}+\Delta \varphi\right)+\Lambda_{0},
\end{aligned}
$$

where $\Lambda_{i}, \Lambda_{0}$ are defined by relations (105), (106). All the constraints Poisson-commute to each other. There are nontrivial involution relations between the constraints and Hamiltonian,

$$
\begin{aligned}
\left\{\tilde{T}_{i j}, H\right\} & =-\left(\delta_{(i}^{k} \partial_{j)}-\frac{1}{d-1} \eta_{i j} \partial^{k}\right) T_{k} \\
\partial_{0} T_{i}+\left\{T_{i}, H\right\} & =-2\left(\delta_{i}^{(j} \partial^{k)}-\frac{1}{d-1} \eta^{j k} \partial_{i}\right) \tilde{T}_{j k}+\partial_{i} T \\
\partial_{0} T+\{T, H\} & =-\frac{1}{d-1} \partial^{i} T_{i} .
\end{aligned}
$$

Once all the constraints are known, and structure coefficients of involution relations (22), (24), (25) are identified, they define the unfree gauge variations of the fields and Lagrange multipliers by the general rules (31), (32). Also, the structure coefficients define Eqs. (33) and (34) imposed on the gauge parameters. Given the constraints (107) and involution relations (108), we apply the general rules and arrive at unfree gauge symmetry transformations of the fields $\varphi_{i j k}, \varphi$ and Lagrange multipliers $\tilde{\varphi}^{i j}$,

$$
\begin{array}{r}
\delta_{\varepsilon} \varphi_{i j k}=3 \partial_{(i} \tilde{\varepsilon}_{j k)}+\frac{3}{d-1} \eta_{(i j} \partial_{k)} \varepsilon, \delta_{\varepsilon} \varphi=3 \partial_{i} \varepsilon^{i}, \\
\delta_{\varepsilon} \tilde{\varphi}^{i j}=\dot{\tilde{\varepsilon}}^{i j}+\partial^{i} \varepsilon^{j}+\partial^{j} \varepsilon^{i}-\frac{2}{d-1} \eta^{i j} \partial_{k} \varepsilon^{k} .
\end{array}
$$

Upon substitution of structure coefficients of involution relations (108) into general relations (33) and (34), we get the constraints on gauge parameters for this model,

$$
\begin{gathered}
\dot{\varepsilon}^{i}+\partial_{j} \tilde{\varepsilon}^{j i}+\frac{1}{d-1} \partial^{i} \varepsilon=0, \\
\dot{\varepsilon}-\partial_{i} \varepsilon^{i}=0 .
\end{gathered}
$$

This unfree gauge symmetry is parametrized by $(d-1)$ tensors $\tilde{\varepsilon}^{i j}, \varepsilon^{i}, \varepsilon$. Explicitly covariant unfree gauge symmetry (87), (88) of the original action (75) is parametrized by symmetric traceless tensor $\varepsilon_{\mu \nu}$. The gauge parameters $\tilde{\varepsilon}^{i j}, \varepsilon^{i}, \varepsilon$ of Hamiltonian form of the symmetry can be viewed as $1+(d-1)$ decomposition of the $d$-tensor parameter $\varepsilon_{\mu \nu}$,

$$
\begin{aligned}
& \varepsilon_{0}^{0}=-\varepsilon^{i}{ }_{i} \equiv-\varepsilon, \quad \varepsilon_{0 i} \equiv \varepsilon_{i}, \\
& \varepsilon_{i j} \equiv \tilde{\varepsilon}_{i j}+\frac{1}{d-1} \eta_{i j} \varepsilon .
\end{aligned}
$$

As we see in this example, the Hamiltonian algorithm of Sec. III allows one to systematically identify all the unfree gauge symmetry transformations and modular parameters of the model, though the method is not explicitly covariant.

\section{E. BFV-BRST formalism for $s=3$ case}

In this subsection, we illustrate the general BFV-BRST formalism of Sec. IV by the spin-3 model (92).

We begin with construction of the formalism by introducing the ghosts of the minimal sector. The ghost pairs are assigned to every constraint of the complete set (107),

$$
\begin{aligned}
\left\{\tilde{C}^{i j}, \tilde{\bar{P}}_{k l}\right\} & =\frac{1}{2}\left(\delta_{k}^{i} \delta_{l}^{j}+\delta_{l}^{i} \delta_{k}^{j}\right)-\frac{1}{d-1} \eta^{i j} \eta_{k l}, \\
\left\{C^{i}, \bar{P}_{i}\right\} & =\delta_{j}^{i}, \quad\{C, \bar{P}\}=1, \\
\operatorname{gh}\left(\tilde{C}^{i j}\right) & =-\operatorname{gh}\left(\tilde{\bar{P}}_{i j}\right)=\operatorname{gh}\left(C^{i}\right) \\
& =-\operatorname{gh}\left(\bar{P}_{i}\right)=\operatorname{gh}(C)=-\operatorname{gh}(\bar{P})=1, \\
\epsilon\left(\tilde{C}^{i j}\right) & =\epsilon\left(\tilde{\bar{P}}_{i j}\right)=\epsilon\left(C^{i}\right) \\
& =\epsilon\left(\bar{P}_{i}\right)=\epsilon(C)=\epsilon(\bar{P})=1 .
\end{aligned}
$$

Hamiltonian BRST generator reads

$Q_{\min }=\tilde{C}^{i j} \tilde{T}_{i j}+C^{i} T_{i}+C T, \quad\left\{Q_{\min }, Q_{\min }\right\}=0$.

Given Hamiltonian $H$ (93), and involution relations (108), the ghost-extended Hamiltonian reads

$$
\begin{gathered}
\mathcal{H}=H-\tilde{C}^{i j} \partial_{(i} \bar{P}_{j)}+C^{i} \partial_{i} \bar{P}-C^{i} \partial^{j} \tilde{\bar{P}}_{j i}-\frac{1}{d-1} C \partial^{i} \bar{P}_{i}, \\
\partial_{0} Q_{\min }+\left\{Q_{\min }, \mathcal{H}\right\}=0 .
\end{gathered}
$$

According to the prescriptions of Sec. IV, the nonminimal sector ghosts are assigned only to the primary constraints 


$$
\begin{aligned}
\left\{\tilde{P}^{i j}, \tilde{\bar{C}}_{k l}\right\} & =\frac{1}{2}\left(\delta_{k}^{i} \delta_{l}^{j}+\delta_{l}^{i} \delta_{k}^{j}\right)-\frac{1}{d-1} \eta^{i j} \eta_{k l}, \\
\operatorname{gh}\left(\tilde{P}^{i j}\right) & =-\operatorname{gh}\left(\tilde{\bar{C}}_{i j}\right)=1, \quad \epsilon\left(\tilde{P}^{i j}\right)=\epsilon\left(\tilde{\bar{C}}_{i j}\right)=1 .
\end{aligned}
$$

Also, the momenta are introduced being canonically conjugate to the Lagrange multipliers,

$$
\begin{aligned}
\left\{\tilde{\varphi}^{i j}, \tilde{\Pi}_{k l}\right\} & =\frac{1}{2}\left(\delta_{k}^{i} \delta_{l}^{j}+\delta_{l}^{i} \delta_{k}^{j}\right)-\frac{1}{d-1} \eta^{i j} \eta_{k l}, \\
\operatorname{gh}\left(\tilde{\varphi}^{i j}\right) & =-\operatorname{gh}\left(\tilde{\Pi}_{i j}\right)=0, \quad \epsilon\left(\tilde{\varphi}^{i j}\right)=\epsilon\left(\tilde{\Pi}_{i j}\right)=0 .
\end{aligned}
$$

Complete Hamiltonian BRST generator reads

$$
Q=Q_{\min }+\tilde{\Pi}_{i j} \tilde{P}^{i j}
$$

Lorentz-like gauge conditions should be imposed, being explicitly resolved with respect to time derivatives of Lagrange multipliers. As explained in Sec. IV, the number of gauges should be the same as the number of primary constraints. So, we choose the following gauges:

$\dot{\tilde{\varphi}}^{i j}-\tilde{\chi}^{i j}=0, \quad \tilde{\chi}^{i j} \equiv-\left(\partial_{k} \varphi^{k i j}-\frac{1}{d-1} \eta^{i j} \partial_{k} \varphi_{l}^{k l}\right)$.

Given the gauges, the gauge Fermion reads

$$
\Psi=\tilde{\bar{C}}_{i j} \tilde{\chi}^{i j}+\tilde{\varphi}^{i j} \tilde{\bar{P}}_{i j} .
$$

Following the general rule (51), the gauge-fixed Hamiltonian is constructed,

$$
\begin{aligned}
H_{\Psi}= & \mathcal{H}+\tilde{\varphi}^{i j} \tilde{T}_{i j}+\tilde{\Pi}_{i j} \tilde{\chi}^{i j}+\tilde{\bar{P}}_{i j} \tilde{P}^{i j}+\tilde{\bar{C}}_{i j}\left\{\tilde{\chi}^{i j}, \tilde{T}_{k l}\right\} \tilde{C}^{k l} \\
& +\tilde{\bar{C}}_{i j}\left\{\tilde{\chi}^{i j}, T_{k}\right\} C^{k}+\tilde{\bar{C}}_{i j}\left\{\tilde{\chi}^{i j}, T\right\} C .
\end{aligned}
$$

As a result, we arrive at the gauge-fixed BRST-invariant Hamiltonian action

$$
\begin{aligned}
S_{\mathrm{BRST}}^{\Psi}= & \int d^{d} x\left[\Pi^{i j k} \dot{\varphi}_{i j k}+\Pi \dot{\varphi}+\tilde{\Pi}_{i j} \dot{\tilde{\varphi}}^{i j}+\tilde{\bar{P}}_{i j} \dot{\tilde{C}}^{i j}\right. \\
& \left.+\bar{P}_{i} \dot{C}^{i}+\bar{P} \dot{C}+\tilde{\bar{C}}_{i j} \dot{\tilde{P}}^{i j}-H_{\Psi}\right] .
\end{aligned}
$$

Corresponding path integral reads

$$
Z_{\Psi}=\int[D \Phi] \exp \left\{\frac{i}{\hbar} S_{\mathrm{BRST}}^{\Psi}\right\}
$$

where $\Phi=\left\{\Pi^{i j k}, \varphi_{i j k}, \Pi, \varphi, \tilde{\Pi}_{i j}, \tilde{\varphi}^{i j}, \tilde{\bar{P}}_{i j}, \tilde{C}^{i j}, \bar{P}_{i}, C^{i}, \bar{P}, C\right.$, $\left.\tilde{\bar{C}}_{i j}, \tilde{P}^{i j}\right\}$. Integration over momenta $\tilde{P}^{i j}, \tilde{\bar{P}}_{i j}, \Pi_{i j k}$, $\Pi$ leads to the following result:

$$
\begin{aligned}
Z= & \int\left[D \Phi^{\prime}\right] \exp \left\{\frac { i } { \hbar } \int d ^ { d } x \left[\mathcal{L}+\tilde{\Pi}_{i j} \partial_{\mu} \varphi^{\mu i j}\right.\right. \\
& \left.\left.+\tilde{\bar{C}}_{i j}\left(\square \delta_{\mu}^{i} \delta_{\nu}^{j}+2 \partial^{i} \delta_{\mu}^{j} \partial_{\nu}\right) C^{\mu \nu}+\bar{P}_{\mu} \partial_{\nu} C^{\nu \mu}\right]\right\},
\end{aligned}
$$

where $\Phi^{\prime}=\left\{\varphi_{\mu \nu \rho}, \tilde{\Pi}_{i j}, \tilde{\bar{C}}_{i j}, C^{\mu \nu}, \bar{P}_{\mu}\right\}$. Ghosts $C, C^{i}, \tilde{C}^{i j}$ can be viewed as $1+(d-1)$ decomposition of ghost $C^{\mu \nu}, C^{\nu}{ }_{\nu}=$ 0 (8), being $d$-dimensional symmetric traceless tensor,

$$
\begin{aligned}
C^{0}{ }_{0} & =-C^{i}{ }_{i} \equiv-C, \quad C_{0 i} \equiv C_{i}, \\
C_{i j} & \equiv \tilde{C}_{i j}+\frac{1}{d-1} \eta_{i j} C .
\end{aligned}
$$

Expression $\partial_{\nu} C^{\nu \mu}$ in the end of exponential of (125) can be viewed as a constraint imposed on the ghosts which corresponds to the transversality condition imposed on gauge parameters. The ghost momenta $\bar{P}_{\mu}: \bar{P}_{0} \equiv-\bar{P}$, assigned to the secondary and tertiary constraints, play the role of Fourier multipliers at the constraints imposed on ghosts for the original unfree gauge symmetry. With this regard, relation (125) is seen to reproduce the modified FP path integral (11), (13) for the original action (75).

\section{CONCLUSION}

In this paper, we work out constrained Hamiltonian formalism corresponding to the unfree gauge symmetry with gauge parameters constrained by differential equations. In the Hamiltonian form, the phenomenon of the unfree gauge symmetry has been clarified from viewpoint of involution relations between Hamiltonian and constraints. The key role is plaid by differential operators $\Gamma$, being the coefficients in the involution relations (22), (24) such that stand at the constraints of the next generation in the stability conditions of the previous constraints. These structure coefficients define the unfree gauge symmetry if they have a finite kernel. Even if $\Gamma$ are nondegenerate (trivial kernel), but the inverse does not exist in the class of differential operators, we have unfree gauge symmetry. Given the structure coefficients of involution relations with these properties, we arrive at Eqs. (33) and (34) constraining the gauge parameters. In the best-known example of the unfree gauge symmetry, the unimodular gravity, the kernel of $\Gamma$ is one-dimensional, and the corresponding modular parameter is the cosmological constant $\Lambda$. The modular parameters are defined by the asymptotics of the fields. For example, even the free spin-2 field theory [1,2] with unfree gauge symmetry in Minkowski space (that corresponds to linearized UG), admits solutions with nonvanishing $\Lambda$. These solutions correspond to nonvanishing fields at infinity. Analogous solutions with nontrivial modular parameters are noticed in Sec. V for higher spin fields with unfree gauge symmetry. The dynamics with nontrivial modular parameters are relevant upon inclusion of interactions as we expect. This issue will be addressed elsewhere. In Sec. IV, we explain how the Hamiltonian 
BFV-BRST formalism is adjusted for the case of unfree gauge symmetry. For the case when there are no higherorder ghost vertices, we deduce from the phase-space path integral the modified FP quantization rules such that account for the unfree gauge symmetry by imposing corresponding constraints on the ghosts. In this way, we see that the covariant quantization rules for the systems with unfree gauge symmetry are deduced from corresponding modification of Hamiltonian BFV-BRST quantization.

\section{ACKNOWLEDGMENTS}

The study of the Hamiltonian structure of unfree gauge symmetry was supported by a government task of the Ministry of Science and Higher Education of the Russian Federation, Project No. 0721-2020-0033. The application of the general formalism to the higher spin models was supported by the Foundation for the Advancement of Theoretical Physics and Mathematics "BASIS".
[1] E. Alvarez, D. Blas, J. Garriga, and E. Verdaguer, Transverse Fierz-Pauli symmetry, Nucl. Phys. B756, 148 (2006).

[2] D. Blas, Gauge symmetry and consistent spin-two theories, J. Phys. A 40, 6965 (2007).

[3] E. D. Skvortsov and M. A. Vasiliev, Transverse invariant higher spin fields, Phys. Lett. B 664, 301 (2008).

[4] A. Campoleoni and D. Francia, Maxwell-like Lagrangians for higher spins, J. High Energy Phys. 03 (2013) 168.

[5] R. Percacci, Unimodular quantum gravity and the cosmological constant, Found. Phys. 48, 1364 (2018).

[6] A. O. Barvinsky and A. Y. Kamenshchik, Darkness without dark matter and energy-generalized unimodular gravity, Phys. Lett. B 774, 59 (2017).

[7] A. O. Barvinsky, N. Kolganov, A. Kurov, and D. Nesterov, Dynamics of the generalized unimodular gravity theory, Phys. Rev. D 100, 023542 (2019).

[8] D. S. Kaparulin and S. L. Lyakhovich, A note on unfree gauge symmetry, Nucl. Phys. B947, 114735 (2019).

[9] D. S. Kaparulin and S. L. Lyakhovich, Unfree gauge symmetry in the BV formalism, Eur. Phys. J. C 79, 718 (2019).

[10] V. A. Abakumova, I. Y. Karataeva, and S. L. Lyakhovich, Unfree gauge symmetry in the Hamiltonian formalism, Phys. Lett. B 802, 135208 (2020).
[11] M. Henneaux and C. Teitelboim, Quantization of Gauge Systems (Princeton University Press, Princeton, NJ, 1992).

[12] D. Francia, G. L. Monaco, and K. Mkrtchyan, Cubic interactions of Maxwell-like higher spins, J. High Energy Phys. 04 (2017) 068.

[13] D. S. Kaparulin, S. L. Lyakhovich, and A. A. Sharapov, Consistent interactions and involution, J. High Energy Phys. 01 (2013) 097.

[14] S. L. Lyakhovich and A. A. Sharapov, Normal forms and gauge symmetries of local dynamics, J. Math. Phys. (N.Y.) 50, 083510 (2009).

[15] D. Francia, S. L. Lyakhovich, and A. A. Sharapov, On the gauge symmetries of Maxwell-like higher-spin Lagrangians, Nucl. Phys. B881, 248 (2014).

[16] I. A. Batalin and S. L. Lyakhovich, Generalized canonical quantization of nonstationary dynamical systems subject to constraints, Proceedings, 18th International Colloquium on Group Theoretical Methods in Physics (GROUP 18), Moscow 1990, Proceedings, Symmetries and Algebraic Structures in Physics (Nova Science Publishers, Inc, 1991), pp. 57-63.

[17] C. Fronsdal, Massless fields with integer spin, Phys. Rev. D 18, 3624 (1978). 